\title{
Simultaneous and synergistic profiling of cloud and drizzle properties using ground-based observations
}

\author{
Stephanie P. Rusli ${ }^{1,2}$, David P. Donovan ${ }^{2}$, and Herman W. J. Russchenberg ${ }^{1}$ \\ ${ }^{1}$ Department of Geoscience and Remote Sensing, Faculty of Civil Engineering and Geosciences, \\ TU Delft, Delft, the Netherlands \\ ${ }^{2}$ Royal Netherlands Meteorological Institute (KNMI), De Bilt, the Netherlands
}

Correspondence to: Stephanie P. Rusli (s.rusli-1@ @udelft.nl)

Received: 9 December 2016 - Discussion started: 2 January 2017

Revised: 2 October 2017 - Accepted: 12 October 2017 - Published: 11 December 2017

\begin{abstract}
Despite the importance of radar reflectivity $(Z)$ measurements in the retrieval of liquid water cloud properties, it remains nontrivial to interpret $Z$ due to the possible presence of drizzle droplets within the clouds. So far, there has been no published work that utilizes $Z$ to identify the presence of drizzle above the cloud base in an optimized and a physically consistent manner. In this work, we develop a retrieval technique that exploits the synergy of different remote sensing systems to carry out this task and to subsequently profile the microphysical properties of the cloud and drizzle in a unified framework. This is accomplished by using ground-based measurements of $Z$, lidar attenuated backscatter below as well as above the cloud base, and microwave brightness temperatures. Fast physical forward models coupled to cloud and drizzle structure parameterization are used in an optimal-estimation-type framework in order to retrieve the best estimate for the cloud and drizzle property profiles. The cloud retrieval is first evaluated using synthetic signals generated from large-eddy simulation (LES) output to verify the forward models used in the retrieval procedure and the vertical parameterization of the liquid water content (LWC). From this exercise it is found that, on average, the cloud properties can be retrieved within $5 \%$ of the mean truth. The full cloud-drizzle retrieval method is then applied to a selected ACCEPT (Analysis of the Composition of Clouds with Extended Polarization Techniques) campaign dataset collected in Cabauw, the Netherlands. An assessment of the retrieval products is performed using three independent methods from the literature; each was specifically developed to retrieve only the cloud properties, the drizzle properties below the cloud base, or the drizzle fraction
\end{abstract}

within the cloud. One-to-one comparisons, taking into account the uncertainties or limitations of each retrieval, show that our results are consistent with what is derived using the three independent methods.

\section{Introduction}

Low-level liquid water clouds are known to have a large areal extent (Hartmann et al., 1992) and consequently a strong impact on the Earth's energy balance (Ramanathan et al., 1989; Slingo, 1990). Observations of these clouds to characterize the microphysical and radiative processes are therefore needed for climate studies. One important aspect of such observations is the presence of drizzle, which is found to be a common occurrence in stratocumulus clouds (Fox and Illingworth, 1997). Drizzle alters the cloud droplet spectra and thus the microphysical structure and radiative properties of the clouds (Feingold et al., 1997; vanZanten et al., 2005). Most notably, drizzle is thought to play a significant role in determining the cloud lifetime (Albrecht, 1989). Additionally, drizzle within the cloud complicates matters by dominating the radar reflectivity $(Z)$ signal. Accurately separating the drizzle contribution from the cloud contribution to the received radar signal is necessary to properly derive the cloud and drizzle properties.

Since liquid water clouds tend to form at relatively low altitudes in the atmosphere, it is easier to observe them from the surface than from space. Ground-based remote sensing systems have the potential to deliver high-resolution timeseries data to evaluate and monitor cloud and drizzle prop- 
erties on a regional scale. A synergistic way of utilizing different remote sensors is a powerful approach that has been widely used to provide a more complete and comprehensive view of these clouds. Active sensors operating in different frequency windows such as radar and lidar provide complementary information on the clouds' vertical structure since they "see" different parts of the cloud (Donovan and van Lammeren, 2001). Microwave radiometers (MWRs) that measure the accumulated radiation along a column provide a particularly accurate way to derive the liquid water path (LWP) of clouds (Westwater, 1978; Peter and Kämpfer, 1992).

Various methods that exploit sensor synergy to profile microphysical properties of the liquid water cloud have been developed (Frisch et al., 1995a; Austin and Stephens, 2001; McFarlane et al., 2002; Löhnert et al., 2001; Brandau et al., 2010; Martucci and O'Dowd, 2011). However, these methods either avoid, ignore, or do not fully capture the presence of drizzle. Other techniques that focus on drizzle retrieval are limited in their application to the region below the cloud base (O'Connor et al., 2005; Westbrook et al., 2010). Retrieving the properties of drizzle that is interspersed within the cloud is indeed more difficult. A few hundred meters into the cloud, lidar backscatter signal no longer carries useful information due to the strong attenuation by cloud droplets. While radar has the capability to penetrate deeper into the cloud, the radar reflectivity is known to be sensitive to drizzle droplets that are larger in size as compared to the cloud droplets. Since the observed reflectivity contains contributions from both cloud and drizzle droplets, its interpretation is not straightforward.

Fielding et al. (2015) set a precedent by jointly retrieving cloud and drizzle properties using ground-based radar, lidar, and Sun-photometer observations. Their retrieval departs from the assumption that drizzle is present only when the maximum observed reflectivity in a given column exceeds a single threshold value. While the existence of such a reflectivity threshold is supported by many observational studies, the empirically determined value differs among these studies and can span quite a wide range. Sauvageot and Omar (1987); Frisch et al. (1995b); Mace and Sassen (2000) suggest different $Z$ thresholds in the range of -20 and $-15 \mathrm{dBZ}$. Baedi et al. (2002) showed that the reflectivity due to a non-drizzle component of the cloud reaches a maximum at about $-20 \mathrm{dBZ}$, while that of the drizzle component is not lower than about $-10 \mathrm{dBZ}$, leaving on average a $\sim 10 \mathrm{~dB}$ reflectivity gap between the drizzle-contaminated and drizzlefree droplet spectrum. Furthermore, Wang and Geerts (2003) demonstrate that the value of this threshold varies with altitude within the cloud layer and it can increase from around $-25 \mathrm{dBZ}$ near the cloud base to about $-12 \mathrm{dBZ}$ close to the cloud top. A theoretical approach by Liu et al. (2008) reveals a dependence of the threshold value on the droplet number concentration - a finding that compares favorably with observations. In remote sensing applications, where droplet concentration is one of the unknown variables to be retrieved, setting a single $Z$ threshold value in advance may lead to an unaccounted bias in the retrieval.

In this work we develop a retrieval technique that combines ground-based radar, lidar, and microwave radiometer measurements to simultaneously profile the cloud and drizzle properties without placing a priori constraints on the presence of drizzle droplets within the cloud. There is no predefined reflectivity threshold and so drizzle is always assumed to be present until that possibility is excluded by the best fit to the data. The MWR brightness temperature and the lidar attenuated backscatter up to a few hundred meters above the cloud base provide much of the constraint on the cloud component, whereas the radar reflectivity is used to then infer the drizzle contribution. Drizzle droplets are set apart from the cloud droplets through the use of a critical effective radius threshold in the algorithm. This choice of threshold is motivated by the recognition that a characteristic or critical droplet radius exists, above which intense droplet coalescence triggers rapid drizzle formation. This radius is found to be $12-14 \mu \mathrm{m}$ as shown by satellite and ground-based observations (Suzuki et al., 2010; Rosenfeld, 2000; Rosenfeld and Gutman, 1994), aircraft measurements (Gerber, 1996; Boers et al., 1998; Freud and Rosenfeld, 2012), and numerical simulations (Magaritz et al., 2009; Pinsky and Khain, 2002; Benmoshe et al., 2012). This retrieval technique allows us to retrieve not only drizzle microphysical properties below the cloud base but also within the cloud at the same time. We apply this algorithm to synthetic signals for a test case, as well as to observational data collected in the fall of 2014 as part of the ACCEPT (Analysis of the Composition of Clouds with Extended Polarization Techniques) field campaign in Cabauw, the Netherlands. The retrieved cloud and drizzle products from the ACCEPT dataset are evaluated against the results of three independent retrieval methods that use the lidar depolarization signal (Donovan et al., 2015), the lidar attenuated backscatter and radar Doppler spectral moments (O'Connor et al., 2005), and the radar Doppler spectra (Kollias et al., 2011a, b; Luke and Kollias, 2013) as their main tools.

The remainder of this paper is organized as follows. Section 2 describes the retrieval procedure in detail, including the theoretical assumptions and the forward models. The test application of this technique to synthetic data based on the large-eddy simulation (LES) output is presented in Sect. 3. In Sect. 4, we perform the cloud and drizzle retrieval on a ground-based dataset. The retrieval products are then evaluated through comparisons with results from three independent retrieval techniques in Sect. 5. To conclude the paper, a summary is provided in Sect. 6 .

\section{Retrieval technique}

The target group for this retrieval technique is single-layered liquid water clouds. Retrieval is not performed when rain 


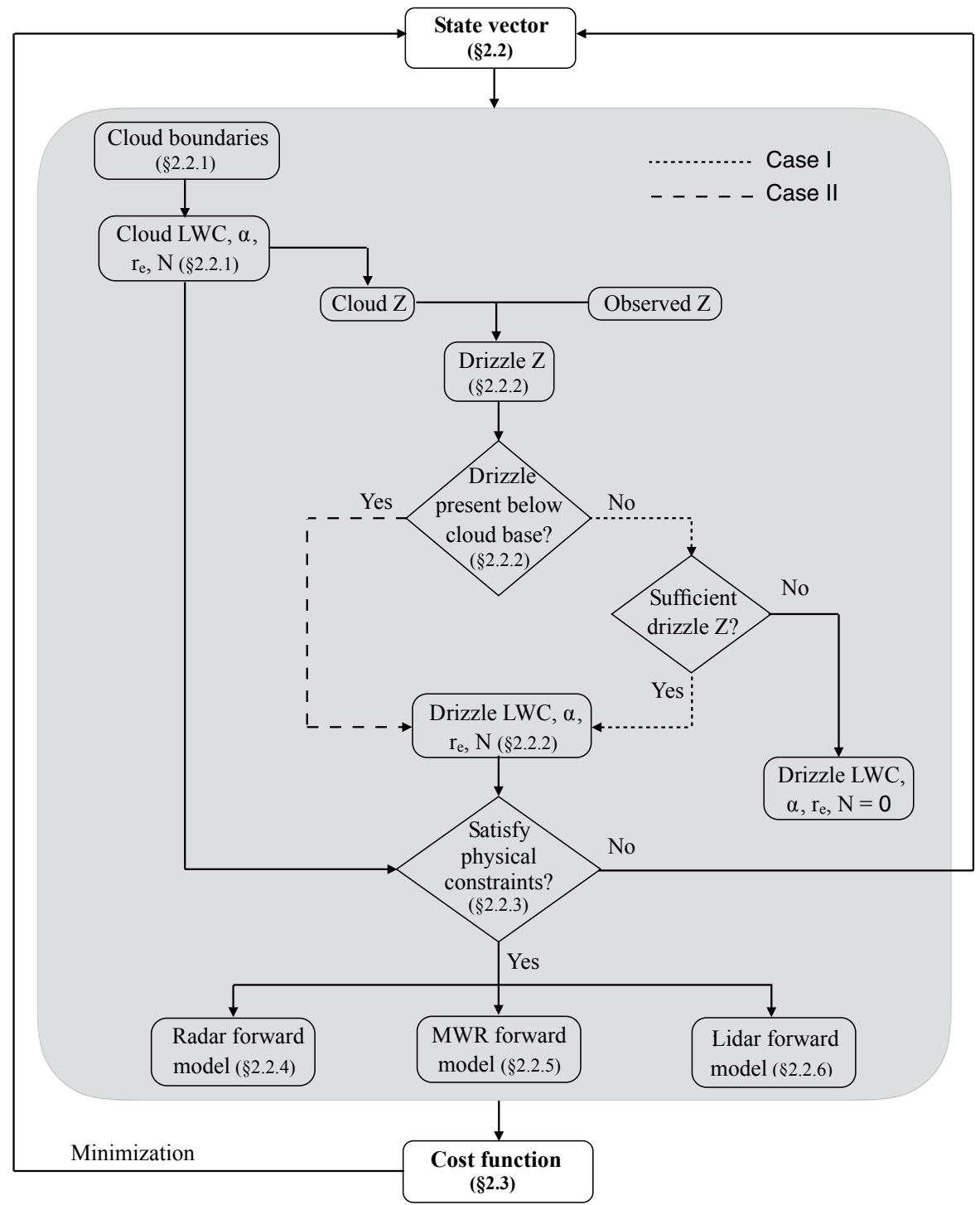

Figure 1. The retrieval flowchart. For each main step, a reference to the section that provides the details is given.

is detected on the ground. The retrieval addresses an inverse problem of deriving the cloud and drizzle profiles that give rise to the observed radar reflectivity $Z$, lidar attenuated backscatter $\beta$, and microwave brightness temperatures $T_{\mathrm{B}}$. The end products include the optical extinction coefficient, liquid water content (LWC), number concentration, and the effective radius of both the cloud and the drizzle components separately, as a function of height.

The overall structure of the retrieval method is depicted in a flowchart in Fig. 1. This flowchart shows the main components of the algorithm, accompanied by references to the relevant sections. Here we give a general overview of how the algorithm works. The state vector refers to the collection of control parameters that we aim to optimize so that our forward models match the observations. These control parame- ters are used to construct cloud and drizzle profiles through the parameterization of the droplet size distributions (DSDs) and their vertical structures outlined in Sect. 2.1. The algorithm starts with determining the cloud boundaries and calculating the cloud microphysical properties (Sect. 2.2.1). It then continues to compute drizzle reflectivities and it proceeds to derive the microphysical properties of drizzle by following one of the two available schemes, depending on whether drizzle is detected below the cloud base (Sect. 2.2.2). If the cloud and drizzle properties satisfy the physical constraints set out in Sect. 2.2.3, the forward models are computed for each instrument (Sect. 2.2.4-2.2.6). These forward models map the theoretical construction of the cloud and drizzle to the observable variables; i.e., they create simulated observations. The cost function quantifies the difference between these pre- 
dicted observations and measured signals. The optimization process to find the best fit solution (Sect. 2.3) to the inverse problem seeks to minimize the cost function. It is worth noting here that this optimization process also accounts for important factors such as lidar calibration uncertainty and the changes in the cloud base height within the measurement temporal resolution.

\subsection{Theoretical basis and parameterization}

\subsubsection{Cloud and drizzle droplet size distribution}

We treat the cloud and the drizzle droplets as two separate entities, and they are assigned independent and unimodal droplet size distribution (DSD) functions, the combination of which results in a bimodal distribution. Here we assume that the number density of the cloud or drizzle droplets as a function of their size can be described by the a generalized gamma distribution (Walko et al., 1995):

$n(r)=\frac{N}{r_{n} \Gamma(v)}\left(\frac{r}{r_{n}}\right)^{v-1} \exp \left(\frac{-r}{r_{n}}\right)$,

where $N=\int_{0}^{\infty} n(r) \mathrm{d} r$ is the total number concentration, $r_{n}$ is the droplet characteristic radius, and $v$ is the shape parameter. The moments of this DSD,

$\left\langle r^{k}\right\rangle=\frac{\int_{0}^{\infty} r^{k} n(r) \mathrm{d} r}{\int_{0}^{\infty} n(r) \mathrm{d} r}$,

are central to defining and deriving the physical properties of the cloud and the drizzle as listed below.

- Effective radius $r_{\mathrm{e}}$ :

$$
r_{\mathrm{e}}=\frac{\left\langle r^{3}\right\rangle}{\left\langle r^{2}\right\rangle}=r_{n}(v+2)
$$

- Extinction coefficient $\alpha$ :

$$
\alpha=\int_{0}^{\infty} Q_{\mathrm{ext}, \lambda}(r) \pi r^{2} n(r) \mathrm{d} r \approx 2 \pi N\left\langle r^{2}\right\rangle,
$$

where the extinction efficiency $Q_{\text {ext }, \lambda}(r) \approx 2$, assuming that the droplets are much larger than the wavelength $\lambda$ of the incident light.

- Liquid water content:

$$
\mathrm{LWC}=\frac{4}{3} \pi \rho_{\mathrm{W}} N\left\langle r^{3}\right\rangle .
$$

In addition, the sixth moment of the distribution function delivers the radar reflectivity factor $Z$ by virtue of Rayleigh approximation, which is valid in the case of scattering of particles whose size is small compared to the radar wavelength. The exact expression is

$Z=2^{6} \int_{0}^{\infty} r^{6} n(r) \mathrm{d} r=64 N\left\langle r^{6}\right\rangle$.

Moreover, the moments of the DSD are assumed to be related to each other such that

$\left\langle r^{a}\right\rangle=k_{\mathrm{ab}}\left\langle r^{b}\right\rangle^{a / b}$,

in which $k_{\mathrm{ab}}$ is a function of shape parameter $v$. Using the property of the gamma function we derive, for instance,

$k_{23}^{3}=\frac{v(v+1)}{(v+2)^{2}}$,

$k_{36}^{2}=\frac{v(v+1)(v+2)}{(v+3)(v+4)(v+5)}$,

which allow one to relate LWC to $\alpha\left(k_{23}\right)$ or $Z\left(k_{36}\right)$.

\subsubsection{Cloud structure}

To profile the cloud, we adopt an approximation for the LWC vertical profile introduced in Boers et al. (2006). Here we repeat what is necessary and adjust some of the notation.

Near the cloud base, LWC is assumed to vary linearly with height (i.e., constant lapse rate). Deeper into the cloud, entrainment leads to a decrease in the LWC lapse rate. The LWC at a given height above the cloud base $\widetilde{z}$ is related to its adiabatic value through a subadiabatic fraction $f(\widetilde{z})$ such that

$\operatorname{LWC}(\widetilde{z})=f(\widetilde{z}) \mathrm{LWC}_{\mathrm{ad}}(\widetilde{z})=f(\widetilde{z}) \rho_{\mathrm{a}} A_{\mathrm{ad}} \widetilde{z}$

$\rho_{\mathrm{W}}$ is the density of water and $\mathrm{LWC}_{\mathrm{ad}}$ the adiabatic LWC. $\rho_{\mathrm{a}}$ and $A_{\mathrm{ad}}$ are the density of air and the adiabatic lapse rate of the liquid water content mixing ratio, respectively; both are a function of the temperature and pressure at the cloud base. The subadiabatic fraction changes as a function of height and is governed by two variables $W$ and $H$ :

$$
\begin{aligned}
f(\widetilde{z}) & =[1-\exp (-W \hat{h})]\left[1-\frac{\exp (-\hat{h}(1-\hat{z}))}{1-\exp (-\hat{h})}\right. \\
& \left.+\frac{\exp (-\hat{h})}{1-\exp (-\hat{h})}\right],
\end{aligned}
$$

where $\hat{h}=x / H$ and $\hat{z}=\tilde{z} / x . x$ is cloud depth, $W$ represents the vertical weight of the liquid water distribution, and the relaxation length scale $H$ indicates how much the liquid water content departs from adiabaticity. The smaller $W$ is, the more 
liquid water there is close to the cloud top. The smaller $H$ is, the closer the actual LWC becomes to the adiabatic profile.

Boers et al. (2006) consider two mixing scenarios to describe the vertical variation in $f(\widetilde{z})$, namely inhomogeneous and homogeneous mixing. Both scenarios are implemented in the retrieval algorithm so the user can decide which one to use. In the first one, the variation of $f(\widetilde{z})$ is attributed to the vertical change in $N$ :

$N(\widetilde{z})=f(\widetilde{z}) N_{\mathrm{ad}}$,

where $N_{\text {ad }}$ is the adiabatic value of $N$. In the homogeneous mixing case, evaporation causes the droplet sizes to decrease while preserving the total number of droplets:

$N(\widetilde{z})=N_{\mathrm{ad}}$

For the retrieval in this paper, we assume for simplicity that $N$ is constant with height. This assumption corresponds to the homogeneous mixing case (Eq. 11).

\subsubsection{Drizzle structure}

The drizzle signature is strongly imprinted in radar reflectivity measurements, making $Z$ indispensable for drizzle retrieval. Owing to the proportionality between the moments of the DSD, the observed reflectivity is related to the drizzle microphysical properties and the vertical shape of $Z$ can be used to profile drizzle. This is especially true below the cloud base where drizzle is isolated from the cloud and $Z$ is related to drizzle alone.

At the very early stages of drizzle formation, when drizzle is still contained within the cloud and there are no detected drizzle droplets falling from the cloud, the analogy of Eq. (9) is used to also describe how the drizzle LWC varies with height. Using Eqs. (4), (5), and (7), $r_{\mathrm{e}}$ can then be written in terms of $v, Z$, and LWC:

$r_{\mathrm{e}}^{3}=\frac{\pi \rho_{z} Z}{48 \mathrm{LWC}} \frac{(v+2)^{3}}{(v+3)(v+4)(v+5))}$,

from which $N$ and $\alpha$ can be computed.

As drizzle starts to grow and leave the cloud, we use a different drizzle parameterization. The vertical profile of the drizzle effective radius above the cloud base is parameterized as a function of height $z$ via an exponential function:

$r_{\mathrm{e}}(z)=r_{\mathrm{e}, \mathrm{cb}} \exp \left(\frac{k_{1}\left(z-z_{\mathrm{cb}}\right)}{z_{\mathrm{dt}}-z_{\mathrm{cb}}}\right)^{-0.5}$.

$r_{\mathrm{e}, \mathrm{cb}}$ is the value of drizzle effective radius at cloud base and $k_{1}$ describes the rate at which $r_{\mathrm{e}}$ decreases towards cloud top. The subscript "dt" denotes drizzle top and "cb" cloud base. The choice of such an exponential function is motivated by the results of in situ drizzle measurements showing that within the cloud the drizzle effective radius displays an exponential-like increase towards the cloud base (Wood, 2005a; Lu et al., 2009).
Below the cloud base, drizzle droplets are not expected to keep growing. Instead, they are assumed to evaporate and shrink. In this region, the parameterization of $r_{\mathrm{e}}$ is based on a simple power law:

$r_{\mathrm{e}}(z)=r_{\mathrm{e}, \mathrm{cb}}\left(\frac{z-z_{\mathrm{db}}}{z_{\mathrm{cb}}-z_{\mathrm{db}}}\right)^{k_{2}}$,

with $k_{2}$ describing the rate at which $r_{\mathrm{e}}$ decreases from the cloud base to the drizzle base (denoted by the subscript "db").

In the retrieval, the two parameters $k_{1}$ and $k_{2}$ are positive and are solved using values of $r_{\mathrm{e}}$ at three different heights: below, at, and above the cloud base (see Sect. 2.2). The droplet size information at the cloud base is crucial since it acts as a scaling factor and is the point where these two functions meet. Once the vertical profiles of $\nu, Z$, and $r_{\mathrm{e}}$ are specified, one can derive LWC, $\alpha$, and $N$ as a function of height.

\subsection{Retrieval scenario}

Following the retrieval flowchart in Fig. 1, we address each of the main steps here. There is a total of 12 elements in the state vector: 7 for the cloud component (Sect. 2.2.1), 4 for the drizzle component (Sect. 2.2.3), and 1 element to compensate for a possible offset in the lidar signal due to imperfect calibration (Sect. 2.2.6). These state vector elements are used throughout the algorithm and their roles are explained below; we mark these 12 parameters with an asterisk (*) to help the reader distinguish them from other variables.

\subsubsection{Cloud profiles}

The profile of cloud LWC is constructed according to Eq. (8), using the subadiabatic fraction $f(\widetilde{z}) . f(\widetilde{z})$, as formulated in Eq. (9), is a function of relative height and depends on three variables: the vertical weight, the relaxation length scale, and the cloud depth. Thus, specifying the cloud LWC profile requires us to determine four parameters: $W_{\text {cld }}^{*}, \hat{h}_{\text {cld }}^{*}$, cloud base height $z_{\mathrm{cb} \text {,opt }}$, and cloud top height $z_{\mathrm{ct} \text { opt }}$. We use lidar and radar observations to get estimates of the cloud base and top altitudes and let the exact locations be optimized in the algorithm. This way, the limited range resolution of the radar and lidar is taken into account in the model. Variables $f t_{\mathrm{cb}}^{*}$ and $f t_{\mathrm{ct}}^{*}$ are employed to serve this purpose - that is, to allow the model cloud boundaries to be located at any spatial point within a given range.

$f t_{\mathrm{ct}}^{*}$ is used to optimize the cloud top height $z_{\mathrm{ct} \text {,opt }}$ based on radar measurements such that

$z_{\mathrm{ct}, \mathrm{opt}}=z_{\mathrm{ct}}+f t_{\mathrm{ct}}^{*} \Delta z_{\mathrm{r}}$.

$\Delta z_{\mathrm{r}}$ is the range resolution of the radar and $z_{\mathrm{ct}}$ is the height of the radar range gate above the last radar detection. The value of $f t_{\mathrm{ct}}^{*}$ is restricted to be in the range $[-1,0]$. 
The cloud base location is estimated from the lidar attenuated backscatter $(\beta)$ profile. There is an added complication that the measured lidar signals appear to have suffered from spatial broadening around the cloud base: the attenuated backscatter increases towards the peak value more mildly than theoretically expected for clouds with a sharp, welldefined boundary, suggesting a somewhat gradual increase in extinction coefficient. This is also true for cases where there is no radar detection below the cloud base. Possible causes for this may include turbulence, entrainment, and changes in cloud base heights within the measurement temporal resolution that blur the cloud boundary, creating a transitional region seen in the lidar signal. This effect is comparatively small in magnitude with respect to the maximum backscatter (see Fig. 2) but would certainly affect the determination of cloud base height and drizzle quantification. We account for this effect by smoothing the cloud LWC around the cloud base, as described in the following paragraphs. Here we first show that this smoothing helps to achieve a better fit. Figure 2 compares the profiles of the forward-modeled $\beta$ with and without LWC smoothing against observational data (circles). Without smoothing, the best fit (forward-modeled) lidar backscatter is amplified strongly within two range gates $(30 \mathrm{~m})$ before it gets attenuated (dashed line). In the height range between 1.20 and $1.26 \mathrm{~km}$, the relative error at the signal peak is the smallest, leading to the good fit at this range gate at the expense of the much worse fit at the earlier range gates. The cloud base height inferred from the model $\beta$ appears to be an overestimate and at this height $\beta$ is underestimated by more than an order of magnitude. When LWC is smoothed around $z_{\mathrm{cb} \text {,opt }}$, the fit improves significantly as shown by the solid line.

To optimize the cloud base height $z_{\mathrm{cb} \text {,opt }}$, we first find the lidar estimate of cloud base height $\left(z_{\mathrm{cb}}\right)$ as the first-order approximation. The lidar attenuated backscatter profile is searched to find the height where $\beta$ reaches its maximum value: $z_{\text {peak }}$. We then identify as $z_{\mathrm{cb}}$ the lowest range gate where $\beta$ rises by more than $50 \%$ to the next range gate and at the same time shows a continuous increase from there up to $z_{\text {peak. }} \cdot z_{\mathrm{cb}, \text { opt }}$ is located somewhere between $z_{\mathrm{cb}}$ and $z_{\text {peak }}$ such that

$z_{\mathrm{cb}, \mathrm{opt}}=z_{\mathrm{cb}}+f t_{\mathrm{cb}}^{*}\left(z_{\mathrm{peak}}-z_{\mathrm{cb}}\right)$,

where $f t_{\mathrm{cb}}$ is constrained to the range $(0,1)$. In practice, when the broadening effect is clearly larger than the lidar range resolution, then one can set the possible range of $f t_{\mathrm{cb}}^{*}$ to $[0,1]$ and write

$z_{\mathrm{cb}, \mathrm{opt}}=z_{\min }+f t_{\mathrm{cb}}^{*}\left(z_{\max }-z_{\min }\right)$.

$z_{\min }$ and $z_{\max }$ are $z_{\mathrm{cb}}+\Delta z_{1}$ and $z_{\text {peak }}-\Delta z_{1}$, respectively, with $\Delta z_{1}$ denoting the lidar range resolution.

Once the cloud LWC profile is set up, the smoothing is applied to the region around $z_{\mathrm{cb} \text {,opt }}$ via the centered moving average scheme. The width of the smoothing window is $2 n+1$,

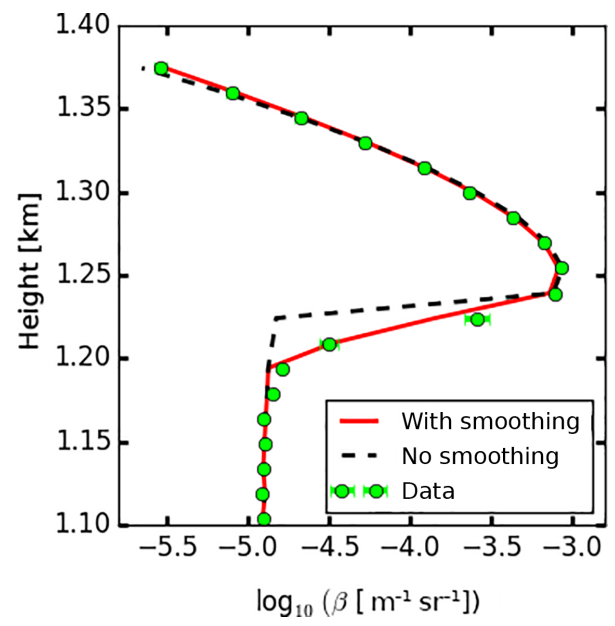

Figure 2. Lidar attenuated backscatter profiles as a function of height. The circles outline an example of a measured $\beta$ profile, taken during the ACCEPT campaign on 26 October 2014 at 5.04 UTC. The dashed black line shows the forward-modeled $\beta$ that best fits the measurements when no smoothing is applied to LWC. The solid red line represents the forward-modeled $\beta$ when LWC is smoothed.

where $n$ is the number of lidar range gates between $z_{\mathrm{cb}}$ and $z_{\mathrm{cb}, \text { opt }}$. The LWC values within the smoothing window are weighted as $\exp \left(-p_{\mathrm{cb}}^{*} d\right) \cdot p_{\mathrm{cb}}^{*}$ acts as a coefficient of the exponential weight and is part of the state vector, whereas $d$ is the distance in the unit of range gates, such that $d=0$ for the central value, $d=1$ for the values next to it, and so on. The smoothing is performed only up to $n+1$ gates above $z_{\mathrm{cb} \text {,opt }}$. Above this height, the impact of the smoothing is insignificant: as $\mathrm{LWC}_{\mathrm{cld}}$ increases up to the peak value in an approximately linear fashion, the effect of the smoothing quickly diminishes with height.

After the smoothed LWC profile is available, $N_{\text {ad }}^{*}$ and $v_{\text {cld }}^{*}$ (both are assumed to be constant with height) given in the state vector can be used to derive profiles of the other cloud properties, i.e., the cloud droplet number concentration (Eq. 10 or 11), the extinction coefficient (Eq. 3), and the effective radius (Eq. 2). In total, seven variables in the state vector are used to construct the profiles of cloud properties: $v_{\mathrm{cld}}^{*}, W_{\mathrm{cld}}^{*}, \hat{h}_{\mathrm{cld}}^{*}, N_{\mathrm{ad}}^{*}, f t_{\mathrm{cb}}^{*}, f t_{\mathrm{ct}}^{*}$, and $p_{\mathrm{cb}}^{*}$.

\subsubsection{Drizzle profile}

From the properties derived in the previous section, the radar reflectivity of the cloud component $Z_{\text {cld }}$ can be computed (Eq. 5). The difference between $Z_{\text {cld }}$ and the observed reflectivity $Z_{\text {obs }}$ is recorded as $Z_{\text {excess }}$ such that

$$
\begin{aligned}
Z_{\text {excess }} & =Z_{\mathrm{obs}}-Z_{\mathrm{cld}} \text { for } Z_{\mathrm{obs}}>Z_{\mathrm{cld}} \\
& =0.0 \text { for } Z_{\mathrm{obs}} \leqslant Z_{\mathrm{cld}} .
\end{aligned}
$$

To enforce some level of spatial continuity for the drizzle above $z_{\mathrm{cb}}$, the resulting $Z_{\text {excess }}$ is smoothed. For simplicity, we apply a simple moving average to $Z_{\text {excess }}$ values within 
cloud boundaries using three range gates as the smoothing width to result in drizzle reflectivity $Z_{\mathrm{dzl}}$. Below the cloud base, $Z_{\mathrm{dzl}}=Z_{\text {excess }}=Z_{\text {obs }}$.

At this point, we can distinguish two types of profiles: with and without radar detection below the cloud base. While radar signal below the cloud base is an obvious sign of the presence of drizzle, its absence, however, does not imply that there are no drizzle droplets within the cloud layers. We classify a profile as non-drizzling when $Z_{\mathrm{dzl}}$ is zero below the cloud base and $Z_{\mathrm{dzl}}>0$ at less than three radar range gates above the cloud base (positive $Z_{\mathrm{dzl}}$ at only one or two gates does not constitute a drizzle profile and is likely dominated by noise). Consequently, the drizzle properties are all set to zero at all heights and the algorithm proceeds to compute the forward models and the cost function considering only contributions from the cloud.

We categorize the drizzling profiles into two cases with different retrieval mechanisms:

- Case I: drizzle is detected only above the cloud base. This case represents very early stages of drizzle formation when the drizzle droplets have not reached below the cloud base and drizzle vertical extent is not known in advance of the retrieval. The absence of a clear, isolated drizzle signature adversely limits the retrieval strategy, so we resort to adopting the same vertical model as for the cloud (Eq. 9). Here, the function $f\left(\widetilde{z}_{\mathrm{d}}\right)$ is defined by $W_{\mathrm{dzl}}^{*}, \hat{h}_{\mathrm{dzl}}^{*}$, with the cloud depth replaced by drizzle depth. The drizzle base and top heights are set to the closest radar range gate beyond the first and last detected $Z_{\mathrm{dzl}}$, respectively, where $Z_{\mathrm{dzl}}=0$. The drizzle LWC profile is thus derived using

$$
\begin{aligned}
\operatorname{LWC}_{\mathrm{dzl}}\left(\widetilde{z}_{\mathrm{d}}\right) & =q_{\mathrm{dzl}}^{*} f\left(\widetilde{z}_{\mathrm{d}}\right) \mathrm{LWC}_{\mathrm{ad}} \\
& =q_{\mathrm{dzl}}^{*} f\left(\widetilde{z}_{\mathrm{d}}\right) \rho_{\mathrm{a}} A_{\mathrm{ad}} \widetilde{z}_{\mathrm{d}} .
\end{aligned}
$$

$\widetilde{z}_{\mathrm{d}}$ is height above the drizzle base while $\mathrm{LWC}_{\mathrm{ad}}$ is identical to that for the cloud, computed using the temperature and pressure at the model cloud base. $q_{\mathrm{dzl}}^{*}$ is a drizzle scaling factor to account for the fact that the adiabatic and drizzle LWC can be a few orders of magnitude apart, a range that is not covered by drizzle $f\left(z_{\mathrm{d}}\right)$ alone.

Given $Z_{\mathrm{dzl}}, \mathrm{LWC}_{\mathrm{dzl}}$, and $v_{\mathrm{dzl}}^{*}$ (assumed independent of height), the profile of the drizzle effective radius can be derived using Eq. (12). The drizzle droplet number concentration and extinction coefficient profiles follow from Eqs. (3) and (4). To sum up, the elements of the state vector used to build the drizzle profile in case I are $v_{\mathrm{dzl}}^{*}, W_{\mathrm{dzl}}^{*}, \hat{h}_{\mathrm{dzl}}^{*}$, and $q_{\mathrm{dzl}}^{*}$.

- Case II: drizzle is detected below and above the cloud base.

The drizzle retrieval here is based on the vertical structure of $r_{\mathrm{e}}$ above (Eq. 13) and below (Eq. 14) the cloud base. The $r_{\mathrm{e}}$ profile is split into the two functional forms to account for the different expected behaviors of drizzle droplets across heights. As in case I, drizzle base and top are set to the closest radar range gate beyond the first and last $Z_{\mathrm{dzl}}$, respectively. This means, to solve Eqs. (13) and (14), we need to determine three parameters: drizzle effective radius at the cloud base, $k_{1}$, and $k_{2}$. In the algorithm, we choose to express these parameters in terms of drizzle extinction coefficients at three height levels: within the cloud, at the cloud base, and below the cloud base. The drizzle effective radius and extinction coefficient are related to each other through $Z_{\mathrm{dzl}}$ and $v_{\mathrm{dzl}}^{*}$. By combining Eqs. (3), (5), and (7), it follows that

$$
r_{e, \mathrm{dzl}}^{4}=\frac{\pi Z_{\mathrm{dzl}}}{32 \alpha_{\mathrm{dzl}}} \frac{\left(v_{\mathrm{dzl}}+2\right)^{3}}{\left(v_{\mathrm{dzl}}+3\right)\left(v_{\mathrm{dzl}}+4\right)\left(v_{\mathrm{dzl}}+5\right)} .
$$

The three extinction coefficients are $\alpha_{\mathrm{id}}^{*}, \alpha_{\mathrm{cb}}^{*}$, and $\alpha_{\mathrm{ic}}^{*}$. The first two refer to drizzle extinction coefficients at the first radar gate (the lowest range gate with radar detection) and at the cloud base. These two variables are used to construct the power-law profile (Eq. 14). At these two heights, $\mathrm{LWC}_{\text {cld }}$ and $\alpha_{\text {cld }}$ are zero, allowing for an unambiguous drizzle retrieval. To specify the drizzle profile above the cloud base, $\alpha_{\mathrm{ic}}^{*}$ is needed. It denotes the drizzle extinction coefficient at a certain height within the cloud. Together with $\alpha_{\mathrm{cb}}^{*}, \alpha_{\mathrm{ic}}^{*}$ solves Eq. (13). The height choice for $\alpha_{\mathrm{ic}}^{*}$ considers the following. The strong attenuation of the lidar signal within the cloud means that useful constraints are available only in the region between cloud base and $\sim 200 \mathrm{~m}$ above it. The drizzle top is usually found at about $200 \mathrm{~m}$ into the cloud or higher. To obtain a meaningful solution for Eq. (13), the height choice for $\alpha_{\mathrm{ic}}^{*}$ should be well above the cloud base. Taking this into account, we opt to retrieve $\alpha_{\mathrm{cb}}^{*}$ at $150 \mathrm{~m}$ above the cloud base.

Having constructed profiles for $Z_{\mathrm{dzl}}$ and the drizzle effective radius, one can easily derive the other microphysical properties with the knowledge of $v_{\mathrm{dzl}}^{*}$. As in case I, $v_{\mathrm{dzl}}^{*}$ is held constant with height. Finally, the four state vector parameters used in case II are $v_{\mathrm{dzl}}^{*}, \alpha_{\mathrm{id}}^{*}, \alpha_{\mathrm{cb}}^{*}$, and $\alpha_{\mathrm{ic}}^{*}$.

\subsubsection{Physical constraints}

Following the above scheme, both the cloud and drizzle properties can be computed for a given state vector. There is, however, no guarantee that these properties are a sensible representation of the system in question. To mitigate this problem we impose several physical constraints that act as filters for the state vector:

- We apply a droplet size threshold to separate the cloud and drizzle regime. It has been shown that there exists a 
critical effective radius between $12-14 \mu \mathrm{m}$, above which coalescence increases and drizzle forms very rapidly (Rosenfeld et al., 2012, and references therein). Based on this, we adopt $13 \mu \mathrm{m}$ as the separation threshold, which means that, at any altitude, the cloud $r_{\mathrm{e}}$ has to be smaller than $13 \mu \mathrm{m}$ and, when drizzle is present, its $r_{\mathrm{e}}$ cannot be less than that. For profiles without radar signal below the cloud base, this threshold plays an important role in categorizing the profile as drizzling or non-drizzling.

- The radar reflectivity due to drizzle must not be higher than the cloud reflectivity near the cloud top. The cloud top region is critical for the cloud-drizzle separation because this region is where the drizzle starts to form and where the difference between cloud and drizzle droplet size is minimal. Since the cloud LWC is highest near the cloud top, it is likely that the cloud number concentration and therefore cloud reflectivity will be dominant here. From this it also follows that the location of the maximum radar reflectivity near the cloud top is an indicator of the location of the $\mathrm{LWC}_{\mathrm{cld}}$ peak.

- For case II, it is important that the drizzle effective radius achieves its maximum value at the cloud base. This follows from the scenario that drizzle droplets grow as they fall through the cloud layers via accretion and then evaporate after they leave the cloud, thereby reducing their size. For case I, the increase in the drizzle effective radius towards the drizzle base is preferred through the use of a penalty function (see Sect. 2.3), but not forced.

- The drizzle effective radius must not be larger than $250 \mu \mathrm{m}$ due to the use of Rayleigh approximation, on which the radar forward model is based. For a $35 \mathrm{GHz}$ radar, the validity of the approximation sets an upper limit of droplet radius at about $280 \mu \mathrm{m}$. The $250 \mu \mathrm{m}$ upper limit is imposed as a safeguard and is more of a technical limitation than a physical one. For the selection of drizzling clouds in our study here, this is not a concern.

\subsubsection{Radar forward model}

Equation (5) relates the cloud or drizzle microphysical properties to the radar reflectivity. The equation assumes the validity of Rayleigh approximation. For comparison with the observed reflectivity, the contribution from the cloud and drizzle must be added and attenuation effects have to be incorporated. The observed reflectivity that we use here is taken the from the Cloudnet categorization product (Illingworth et al., 2007) and has been corrected for two-way attenuation due to atmospheric gases and, in some cases, liquid water. Since the liquid water attenuation is dependent on the availability or the reliability of liquid water path measurements, it is not consistently applied to radar pixels containing cloud and/or drizzle droplets in the Cloudnet algorithm. For this reason, we recover the measured reflectivity before the liquid, but after the gas, the attenuation correction $Z_{\text {obs }}$ using the information provided in the same Cloudnet product. The liquid attenuation is then incorporated in the forward model to compute

$Z_{\mathrm{fm}}=\left(Z_{\mathrm{cld}}+Z_{\mathrm{dzl}}\right) \exp (-2 \tau)$.

Hereinafter, the subscripts "cld" and "dzl" refer to the cloud and the drizzle components, respectively. The optical depth $\tau$ is calculated from the cloud and drizzle LWC using the approximation for the attenuation coefficient given in Liebe et al. (1989). $Z_{\mathrm{fm}}$ is compared to $Z_{\mathrm{obs}}$ during the fitting in the retrieval.

\subsubsection{MWR forward model}

To simulate microwave brightness temperatures $T_{\mathrm{B}}$, gaseous absorption by water vapor and oxygen is computed according to Rosenkranz (1998) and the absorption due to liquid water according to Liebe et al. (1993). The forward radiative transfer calculation is then performed by integrating the radiation intensity along the vertical path up to an altitude of $30 \mathrm{~km}$, neglecting the variation in optical depth due to scattering. As such, $T_{\mathrm{B}}$ measurements provide constraints on the liquid water path of a given column. This forward model also requires additional information on the pressure, temperature, and humidity profiles up to $30 \mathrm{~km}$, which can be obtained from a numerical forecast model or radiosonde data.

\subsubsection{Lidar forward model}

A publicly available code ${ }^{1}$ for the calculation of lidar signals including multiple scattering is used to simulate the lidar attenuated backscatter. In treating the multiple scattering, the code allows an explicit computation of higher scattering orders following an approach by Eloranta (1998) and a fast calculation using the photon variance-covariance method (Hogan, 2006, 2008). Once the relevant parameters are available, e.g., lidar setup, extinction coefficient, and droplet size profiles, lidar attenuated backscatter from below and within the cloud can be calculated.

In the retrieval, we attempt to reconstruct the attenuated backscatter profile in the cloudy, drizzling regions as well as at drizzle-free altitudes between the ground and the cloud. The latter is necessary to estimate a possible offset in lidar calibration. At these altitudes, scattering due to air molecules and aerosol particles is expected to prevail. While it is straightforward to approximate the extinction coefficient due to air molecules $\alpha_{\mathrm{m}}$ from the temperature and pressure profile, the aerosol extinction coefficient $\alpha_{\mathrm{a}}$ is largely unknown. Since multiple scattering does not play an important role in this region below the cloud or drizzle base, we

\footnotetext{
${ }^{1}$ http://www.met.reading.ac.uk/clouds/multiscatter/
} 
use Klett inversion for a two-component atmosphere (Klett, 1981; Kovalev, 1995) to infer $\alpha_{\mathrm{a}}$ from the observed $\beta$ profile such that for $z<z_{0}$

$\alpha^{\prime}(z)=\left[\frac{\left(\frac{P^{\prime}(z) z^{2}}{P^{\prime}\left(z_{0}\right) z_{0}^{2}}\right)}{\frac{1}{\alpha^{\prime *} 0}+2 \int_{z}^{z_{0}} \frac{P^{\prime}\left(z^{\prime}\right) z^{2}}{P^{\prime}\left(z_{0}\right) z_{0}^{2}} \mathrm{~d} z^{\varsigma}}\right]$,

where

$\alpha^{\prime}(z)=\alpha_{\mathrm{a}}(z)+S_{\mathrm{a}} \beta_{\mathrm{m}}(z)$,

$P^{\prime}(z)=S_{\mathrm{a}} P(z) \exp \left(2 \int_{0}^{z}\left(\alpha_{\mathrm{m}}\left(z^{\circ}\right)+S_{\mathrm{a}} \beta_{\mathrm{m}}\left(z^{\circ}\right)\right) \mathrm{d} z^{\circ}\right)$.

The zero subscript refers to the Klett reference point where $z_{0}$ is set to $\min \left(z_{\mathrm{cb}}, z_{\mathrm{db}}\right)$ and $\alpha^{\prime}\left(z_{0}\right)$ is equivalent to $\alpha_{0}{ }^{\prime *}$, one of the state vector elements. $S_{\mathrm{a}}$ is the extinction-tobackscatter ratio for aerosol. Given that the lidar operates at $355 \mathrm{~nm}$, we adopt $S_{\mathrm{a}}=50 \mathrm{sr}$, which is a representative value for aerosol particles. $\beta_{\mathrm{m}}(z)=\alpha_{\mathrm{m}}(z) / S_{\mathrm{m}}$ is the attenuated backscatter due to molecular scattering and is calculated assuming $S_{\mathrm{m}}=8 \pi / 3 . P(z)$ is the attenuated backscatter power as a function of height and is defined as

$P(z)=\frac{C_{\mathrm{ldr}}\left(\beta_{z}(z)+\beta_{\mathrm{m}}(z)\right)}{z^{2} \exp \left(2 \int_{0}^{z}\left(\alpha_{\mathrm{a}}\left(z^{6}\right)+\alpha_{\mathrm{m}}\left(z^{6}\right)\right) \mathrm{d} z^{6}\right)}$.

Using the equations above, the $\alpha_{0}{ }^{\prime *}$ value in the state vector, and the fact that the lidar calibration factor $C_{\text {ldr }}$ cancels out in Eq. (23), the $\alpha_{\mathrm{a}}$ vertical profile below the reference point can be derived. The $\alpha_{\mathrm{a}}(z)$, the drizzle, and the cloud extinction coefficient profiles (as derived in Sect. 2.2.1 and 2.2.2) are then stitched together and used as input for the multiple scattering code to construct the complete attenuated backscatter profile below and within the cloud and drizzle. The lidar calibration factor $C_{\mathrm{ldr}}$ can now be computed at each range gate in the cloud- and drizzle-free region:

$C_{\mathrm{ldr}}(z)=\frac{S_{\mathrm{a}} \beta_{\mathrm{obs}}(z) \exp \left(2 \int_{0}^{z}\left(\alpha_{\mathrm{a}}\left(z^{\circ}\right)+\alpha_{\mathrm{m}}\left(z^{\circ}\right)\right) \mathrm{d} z^{\circ}\right)}{\alpha^{\prime}(z)}$.

Since $C_{\mathrm{ldr}}$ serves to compensate for a systematic offset due to inaccurate calibration, its values are expected to be approximately constant with heights. We have confirmed that the values are very similar across heights to within about $2 \%$. Finally, we multiply the forward-modeled $\beta$ profile by the median value of $C_{\mathrm{ldr}}(z)$ for comparison with the observed $\beta$.

\subsection{Finding the optimal solution}

This retrieval procedure attempts to solve the inverse problem of deriving cloud and drizzle properties from observations by minimizing the cost function

$c f=[y-F(x)]^{T} S_{y}^{-1}[y-F(x)]$ to arrive at the optimal solution for the state vector $x . y$ is the measurement vector defined as

$$
\begin{aligned}
& y=\left[T_{B, \mathrm{obs}, 1}, \ldots T_{B, \mathrm{obs}, \mathrm{nf}}, \beta_{\mathrm{obs}, 1}, \ldots, \beta_{\mathrm{obs}, \mathrm{nl}}, Z_{\mathrm{obs}, 1},\right. \\
& \ldots ., Z_{\mathrm{obs}, \mathrm{nr}},
\end{aligned}
$$

with nf, nl, and nr representing the number of MWR frequency channels (14), the number of lidar range gates, and the number of radar range gates with detection, respectively. $F(x)$ is the vector of forward-modeled observable variables, with the same composition as $y$. For a $T_{\mathrm{B}}$ measurement at a frequency $i$ with an uncertainty $\sigma_{T_{\mathrm{B}}, i}$, the diagonal element $(i, i)$ of the measurement covariance matrix $S_{y}$ is $\sigma_{T_{\mathrm{B}}, i}^{2}$. All non-diagonal elements (cross-channel or cross-instrument elements) are set to zero assuming no correlation. The elements $(m, n)$ of matrix $S_{y}$ corresponding to radar and lidar data are calculated according to

$S_{y, m, n}=E\left(\left[y_{m}-E\left(y_{m}\right)\right]\left[y_{n}-E\left(y_{n}\right)\right]\right)$,

which results in

$$
\begin{aligned}
S_{y, m, n} & =\sigma_{C}^{2} y_{m} y_{n} \text { for } m \neq n, \\
& =\sigma_{C}^{2} y_{m}^{2}+\sigma_{y_{\mathrm{m}}}^{2} \text { for } m=n,
\end{aligned}
$$

where $\sigma_{y_{\mathrm{m}}}$ and $\sigma_{C}$ are the random uncertainties of the measured signal and the instrument calibration, respectively. $\sigma_{C}$ is set to be small (comparable to the desired fit accuracy for $Z$ and $\beta$ ).

For case I, a penalty term is added to the cost function to bias $r_{e, \mathrm{dzl}}$ toward the desired profile. Along the drizzle profile, it is checked whether $r_{e, \text { dzl }}$ is larger than the one directly below it. Since the determining factor of drizzle is the radar reflectivity, the penalty is applied to the radar part of the cost function such that every single violation would add the radar term to the original cost function.

The cost function (combined with the penalty function for case I) is minimized using differential evolution (DE), a global stochastic optimization technique similar to population-based optimization routines (Storn and Price, 1997). DE does not require gradient information, which is an advantage given the complexity and the nonlinearity of the cost function. It is designed to deliver robust results and a fast convergence while maintaining a small number of control variables. This minimization algorithm begins with a population of state vectors that constitute a generation. The vector values are chosen to cover the allowed parameter space and the population is then updated with each generation. For each member vector, a new vector is created through mutation and parameter mixing/crossover to replace the old one if it results in a smaller cost function value. Otherwise, the old member vector is retained to be part of the subsequent generation population. This mutation-crossover scheme, along with the strategy to start with a set of vectors instead of a single initial vector, makes it less likely for the algorithm to get trapped in local minima. To use the algorithm, initial guesses 
Table 1. Lower and upper bounds for the state vector values used in the optimization. The units, when relevant, are given in the square brackets.

\begin{tabular}{lrr}
\hline State vector element & Lower limit & Upper limit \\
\hline$v_{\mathrm{cld}}$ & 2 & 20 \\
$\hat{h}$ (cloud and drizzle) & 0.001 & 35 \\
$W$ (cloud and drizzle) & 0.001 & 1 \\
$N_{\mathrm{ad}}\left(\right.$ cloud; $\left.\left[\mathrm{m}^{-3}\right]\right)$ & $10^{7}$ & $5 \times 10^{9}$ \\
$f t_{\mathrm{cb}}$ & 0 & 1 \\
$f t_{\mathrm{ct}}$ & -1 & 0 \\
$v_{\mathrm{dzl}}$ & 1 & 10 \\
$q$ & 0.001 & 0.03 \\
$\alpha_{\mathrm{ic}}\left[\alpha_{\mathrm{cld}}\right]$ & $10^{-5}$ & $10^{-2}$ \\
$\alpha_{\mathrm{cb}}\left[m^{-1}\right]$ & $10^{-6}$ & $10^{-4}$ \\
$\alpha_{\mathrm{id}}\left[\alpha_{\mathrm{cb}}\right]$ & 0.001 & 1 \\
$\alpha_{0}^{\prime}\left[\mathrm{m}^{-1}\right]$ & $10^{-10}$ & $10^{-3}$ \\
$p_{\mathrm{cb}}$ & 1 & 3 \\
\hline
\end{tabular}

for the state vector values are not needed, but the lower and upper limits for each state vector parameter are required.

DE comes in several variants, which differ in the way the mutation and crossover are done. Here we choose the $\mathrm{DE} /$ best/1/bin variant with a population size $\mathrm{NP}=10$, a mutation factor $\mathrm{F}$ that randomly changes between 0 and 1.9 on a generation-by-generation basis, and a crossover constant $\mathrm{CR}=0.8$ (Storn and Price, 1997). For the retrieval, we use the numerical implementation of DE provided within the Python-based environment for scientific computing $\mathrm{SciPy}^{2}$, where the stop conditions are specified by the tolerance (0.01) and the maximum number of generations (150).

The minimization of the cost function is performed over bounded state vector values, from which a physically sensible solution should be found. Unless stated otherwise, the lower and upper limits of the state vector values that we use in this work are listed in Table 1. The shape parameter for the cloud DSD is expected to vary between 2 and 10 (Miles et al., 2000; Gonçalves et al., 2008), depending on, for example, air mass and location (marine or continental). For drizzle DSD, an exponential fit $(v=1)$ is found to be a good approximation (Wood, 2005b). Here we allow $v$ to vary within a wide range. From our investigation (Sect. 3) it appears that constraining $v$ to a fixed value when the radar calibration accuracy is unknown can potentially create a significant bias in the retrieval products. The limits for $\hat{h}$ and $W$ cover the subadiabatic range of LWP that is viewed to be common (Boers et al., 2006). Since small drizzle droplets present minimal effects on $\beta$, the extinction coefficient of drizzle at the cloud base $\alpha_{\mathrm{cb}}$ is constrained to be comparable to the air extinction coefficient. The value of $\alpha_{\mathrm{id}}$ is expressed relative to the drizzle extinction coefficient at the cloud base and $\alpha_{\text {ic }}$ is determined relative to the value of the cloud extinction coefficient

\footnotetext{
${ }^{2}$ http://www.scipy.org
}

value at the same height. $\alpha_{0}^{\prime}$ is given a large range because it is rather sensitive to a small change in the retrieved lidar offset.

The uncertainties for the optimal solution are computed using Monte Carlo realizations that were generated by perturbing the observations. Each random realization of the observations is drawn from a Gaussian distribution centered on the measurements with the dispersion taken from the measurement (random) errors. The retrieval procedure is then performed on all realizations resulting in a set of solutions. The RMS difference with the optimal solution is calculated to represent the uncertainties of the retrieval. For each column observation, we create 10 realizations which should provide a conservative estimate of the random uncertainty. Systematic uncertainties due to inaccurate radar calibration are not included in the Monte Carlo error estimate. Assuming that the calibration offset can be under- or overestimated by up to a factor of $2(3 \mathrm{~dB})$, the resulting systematic errors on the retrieval products are found to be larger than the random uncertainties (see Sect. 3).

\section{Test using synthetic data}

\subsection{Cloud retrieval}

Before applying the technique to real observational data we test it on a set of synthetic signals generated from large-eddy simulation results. Similar to the work described in Donovan et al. (2015), the simulation setup is based on output from the Dutch Atmospheric LES model (DALES; Heus et al., 2010) for conditions corresponding to the FIRE campaign (Duynkerke et al., 2004; de Roode and Los, 2008). Given the LWC from the LES, the DSD is assumed to be a monomodal gamma distribution, i.e., drizzle droplets are not present. The shape parameter and the number concentration along the vertical column are externally imposed and they are largely constant. ECSIM (Voors et al., 2007; Donovan et al., 2015) was used to generate the radar, lidar, and MWR signals. Applying the algorithm to these signals serves primarily as a sanity check for the retrieval code to verify the forward models and the assumption on the vertical shape of LWC.

The synthetic signals are simulated for a zenith-pointing $32 \mathrm{GHz}$ radar, a lidar operating at $353 \mathrm{~nm}$, and an MWR with 14 frequency channels between 20 and $60 \mathrm{GHz}$ to mimic the instruments used in the ACCEPT campaign (see Sect. 4). The radar and lidar signals are sampled at a fine spatial resolution: $2.5 \mathrm{~m}$ vertically and $25 \mathrm{~m}$ horizontally. To mimic real observations we degrade the vertical resolution of both the radar and lidar to $22.5 \mathrm{~m}$. Along the horizontal axis (corresponding to the time axis), we lower the resolution to $150 \mathrm{~m}$ by averaging radar reflectivity and lidar attenuated backscatter data at each range gate and by averaging the brightness temperatures at each frequency channel. The standard deviation of the mean serves as the uncertainty. The atmosphere be- 
low the cloud is rather static, making the standard deviation of $\beta$ in this region unrealistically low. This condition virtually assigns a lot of weight to the part below the cloud in the fitting process, which leads to inaccurate retrieval. For this reason, we set the noise floor for the $\beta$ profile to $1 \%$ below the cloud base and $5 \%$ above the cloud base. Similarly for $Z$ and $T_{\mathrm{B}}$, the minimum relative error is set to 0.03 and 0.01 , respectively. It is these simulated measurements with adjusted resolution, together with the uncertainties, that are fed to the retrieval code.

Figure 3 shows the input synthetic signals as compared to the signals recovered by the retrieval. Apart from the lower edge of the cloud at horizontal distances $<7.5, Z$ is generally well reproduced. The lidar signal is also recovered despite the noise. It is fitted up to $300 \mathrm{~m}$ into the cloud, after which the noise prevails. The histograms of the reflectivity and the attenuated backscatter residuals (truth minus retrieval) are displayed in Fig. 3e and f. Most of the residuals are relatively small, the peaks of the histograms are centered at zero, and the distributions are quite symmetric with no particularly strong tendency towards positive or negative values. $T_{\mathrm{B}}$, averaged over distance, at each frequency channel coincides well with the data. Average $T_{\mathrm{B}}$ fluctuations over time are small: less than $4 \%$ as shown in Fig. 3f. The root-meansquare deviation (RMSD) between the data and the retrieval is also very low, i.e., less than $1 \%$, suggesting a good match between the two. The maximum RMS is found at $31.4 \mathrm{GHz}$, where the extinction due to liquid water dominates the microwave signal.

Figure 4 displays the true microphysical and optical properties in comparison with the retrieved ones. The structures in the $\mathrm{LWC}, r_{\mathrm{e}}$, and $\alpha$ are mostly reproduced. Since the retrieval is performed on a column-by-column basis the retrieval is not entirely smooth along the horizontal axis and this effect is particularly visible in $N$. The mild vertical structure in the true droplet concentration is not reproduced due to the model assumption of constant $N$. From the histograms, it can be seen that generally the LWC and the extinction coefficient are retrieved more accurately than the effective radius and the number concentration. The retrieved $r_{\mathrm{e}}$ and $N$ tend to be higher and lower than the truth, respectively, by a few percents (see also Fig. 5 and Table 2). The distribution of $\Delta N$ appears less Gaussian than those for the other microphysical properties due to the column gradient in the true $N$ that is not matched by the retrieval assumption.

Figure 5 displays the vertically collapsed version of Fig. 4. LWC and $\alpha$ are integrated into LWP and optical depth, respectively. $N$ and $r_{\mathrm{e}}$ are vertically averaged, with the latter weighted by $\alpha$. The error bars represent the random measurement error from the Monte Carlo realizations; there is no systematic error due to the radar calibration. The fluctuations of the variables along the horizontal axis are easily reproduced with very little bias, which is mostly seen in $r_{\mathrm{e}}$ and $N$, as shown before by the histograms in Fig. 4 . The mean values of the LWP, $r_{\mathrm{e}}$, optical depth, and the number concentration, averaged over the horizontal axis, and the deviation from the truth are given in Table 2.

The true shape parameter is not strictly constant along the vertical direction; it is mostly close to 6 and decreasing to around 2 at the cloud base or cloud top. The retrieval is performed with $v=5.5$ and with the radar calibration factor fixed to 1 to match the true values. The lidar calibration factor is retrieved on average with a $5 \%$ accuracy. For comparison purposes, we also include in the last column of Table 2 the run where the shape parameter $v$ is free within a fixed range, i.e., between 2 and 10 . The result is that the noise of the retrieved products increases but there is very little systematic offset. The optimized $v$ is found to have a mean of 5.98 (RMSD $=2.01)$, which is very close to the true $v$. By comparing the last two columns in Table 2, it is apparent that when $v$ is not fixed the RMSD increases significantly due to the large column-to-column fluctuation but the mean values are hardly affected. The extinction coefficient is found to be relatively stable against the variation in $v$, possibly because its retrieval is largely dependent on the $\beta$ profile.

We also investigate the effect of under- or overestimating the radar calibration offset. For this purpose, we apply a shift of $\pm 3 \mathrm{~dB}$ (a factor of 2 ) to the forward-modeled $Z$ and perform the retrieval with $v$ bounded between 2 and 10 . When the offset is underestimated (forward-modeled $Z$ is multiplied by 0.5 , or $\left.C_{\mathrm{r}}=0.5\right)$, LWC and $r_{\mathrm{e}}$ are overestimated by 10-15\% while $\alpha$ generally becomes lower by a few percent, and vice versa when $C_{\mathrm{r}}=2$. The retrieved number concentration tends to fluctuate and is on average $15 \%$ higher than the mean truth for $C_{\mathrm{r}}=2$. The relatively mild systematic impact of doubling or halving the radar calibration offset is possibly due to the fact that the shape parameter is allowed to vary within a certain range; the true shape parameter is not recovered in both cases of $C_{\mathrm{r}}$. The magnitude of the systematic difference between the retrieval products and the truth increases when $v$ is fixed to the true value, especially for the number concentration where the mean retrieved $N$ becomes $36 \%$ lower than the truth.

What is demonstrated with this exercise is that the forward models are able to reproduce the radar, lidar, and MWR signals and that the LWC parameterization that we use for the cloud indeed provides a realistic description of LWC vertical structure. Given accurate instrument calibration, the systematic mismatch between the retrieval and the truth is found to be very small for this test case, both when $v$ is fixed to approximately the true value or when it is optimized. From all four retrieval products, the largest mean offset from the truth is found for the number concentration $N$ at less than $5 \%$.

\subsection{Cloud and drizzle retrieval}

In this section we present two examples of the cloud and drizzle retrieval using synthetic data to illustrate the drizzle retrieval scheme described in Sect. 2.2.2. The synthetic data are produced as follows. Cloud LWC is provided by 

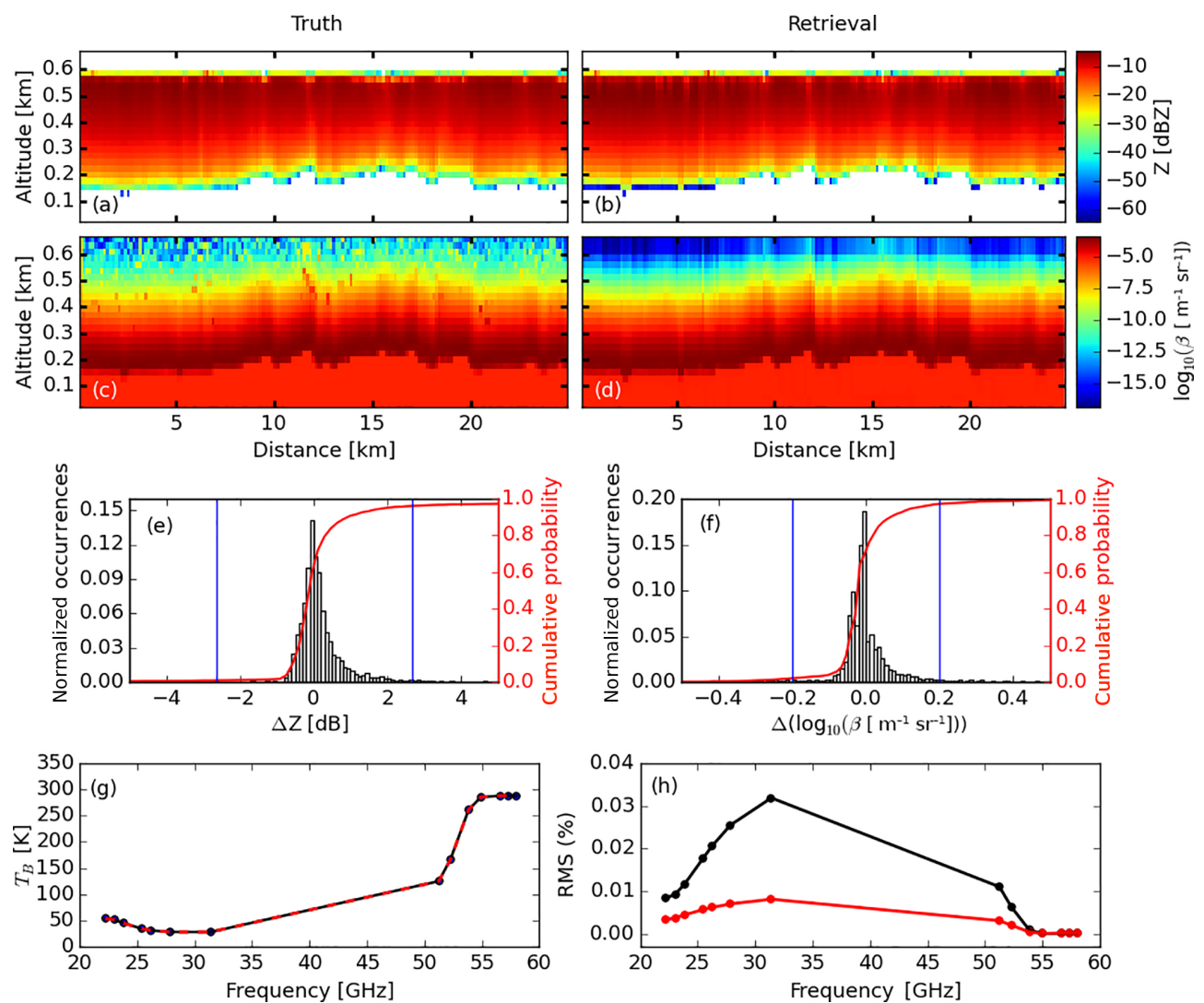

Figure 3. Synthetic signals generated using ECSIM based on LES. Panels (a)-(d) compare radar reflectivity $Z$ and lidar attenuated backscatter $\beta$ between the synthetic signals that are fed to the retrieval as input $(\mathbf{a}, \mathbf{c})$ and best fit produced by the retrieval $(\mathbf{b}, \mathbf{d})$. Panels $(\mathbf{e})$ and (f): histograms of the differences between the true and the retrieved signals (truth minus retrieval). The spread of the distribution is indicated by the blue vertical lines that mark the interval within which $95 \%$ of the total occurrences are found. The red curves show the cumulative distributions. Panel (g) shows brightness temperatures $T_{\mathrm{B}}$ averaged over distance at each frequency channel: the black circles and line show the synthetic measurements (data mean) while the dashed red line shows the retrieval mean. In (h) we plot the standard deviation of the data mean (black line in $\mathbf{g}$ ) divided by the data mean itself (black) and the RMSD between the retrieval and the data divided by the data mean (red).

Table 2. Values of the LWP, $r_{\mathrm{e}}$, optical depth, and the number concentration (as shown in Fig. 5), averaged over the horizontal distance. The RMSD between the truth and the retrieved values is given as the error of the retrievals. The last column is given here for completeness - see discussion in the text.

\begin{tabular}{lrrr}
\hline & Truth & Retrieval with $v=5.5$ & Retrieval with optimized $v$ \\
\hline LWP $\left(\mathrm{g} \mathrm{m}^{-2}\right)$ & 171.68 & $171.96 \pm 5.31$ & $171.64 \pm 9.60$ \\
Effective radius $(\mu \mathrm{m})$ & 20.26 & $20.39 \pm 0.23$ & $20.44 \pm 0.84$ \\
Optical depth & 12.68 & $12.62 \pm 0.46$ & $12.56 \pm 0.45$ \\
Number concentration $\left(\mathrm{cm}^{-3}\right)$ & 21.26 & $20.30 \pm 1.47$ & $20.83 \pm 5.14$ \\
\hline
\end{tabular}

the LES results as in Sect. 3.1. We select one LWC profile and derive the effective radius and extinction coefficient profiles by imposing a monomodal gamma DSD and a value for the number concentration. The DSD shape parameter and the number concentration are set to be independent of height. Two idealized drizzle profiles for both case I and case II are then constructed following the parameterizations introduced in Sect. 2.1.3. These cloud and drizzle profiles serve as the truth to which the retrieval products are later compared against. The two drizzle truths are combined with the cloud truth to result in two example profiles, which are then forward modeled to produce synthetic radar, lidar, and MWR signals. The truths and the retrieval results for the cloud and drizzle components representing the two retrieval cases are displayed in Fig. 6, which is discussed below. 

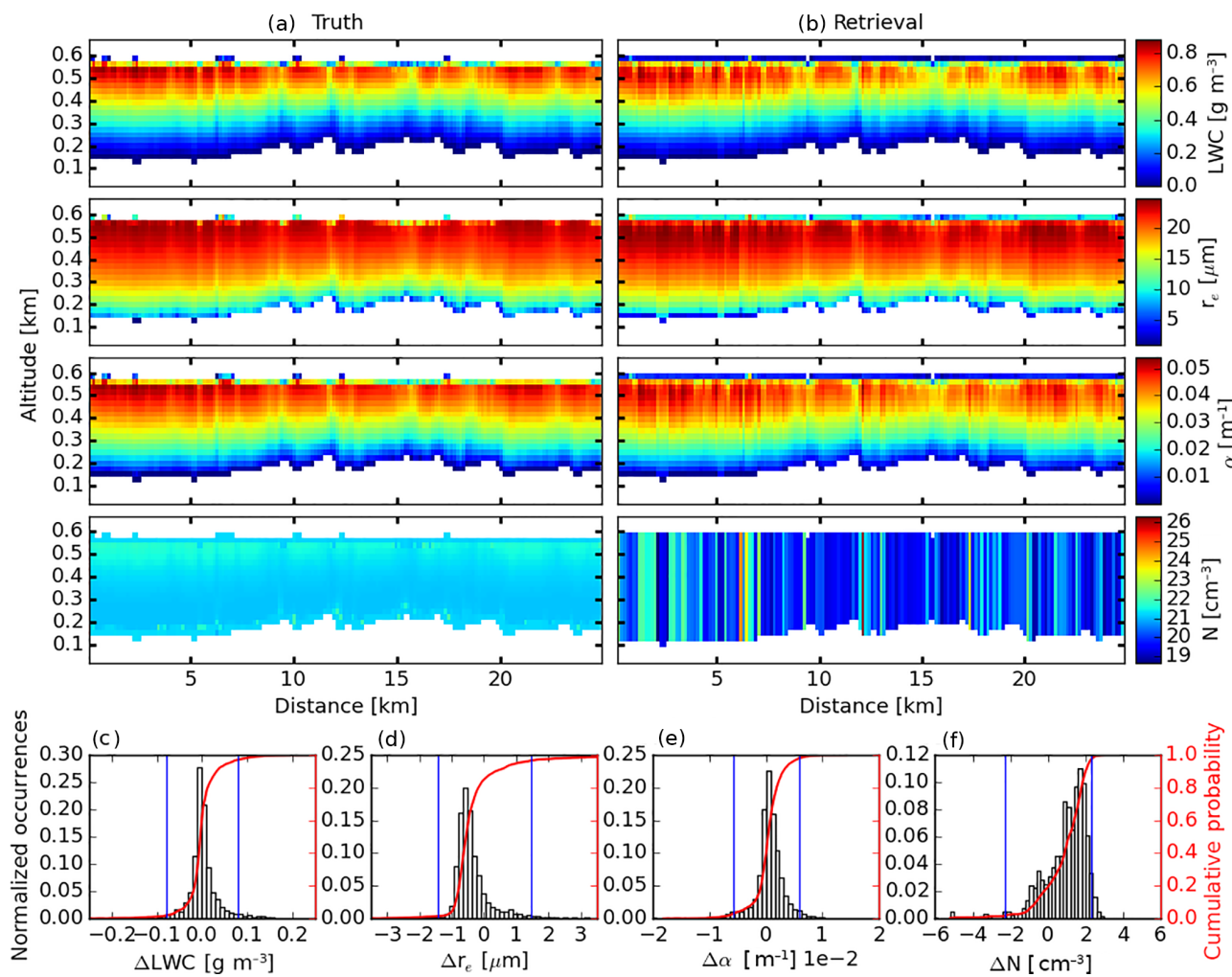

Figure 4. True (a) and retrieved (b) microphysical and optical properties corresponding to the synthetic signal shown in Fig. 3 as a function of vertical and horizontal distance. From top to bottom: liquid water content, effective radius, optical extinction coefficient, and number concentration. The last row (c-f): histograms of the differences between the true and the retrieved cloud properties (truth minus retrieved). The spread of the distribution is indicated by the blue vertical lines that mark the interval within which $95 \%$ of the total occurrences are found. The right $y$ axes of the four histograms are all identical and correspond to the cumulative distributions shown by the red curves.

- Case I

This is the case where drizzle presence is restricted to the cloudy region. There is only a limited amount of information that we can use to retrieve the cloud and drizzle properties in such a situation. Since drizzle contributes only to a small fraction of the total signals, the task of retrieving its properties becomes even more challenging. For the drizzle properties, our goal here is to get an order-of-magnitude estimate rather than to achieve a high accuracy.

In the example here, drizzle is added to all gates within the cloud. Panels Ie and Ii (Fig. 6) show the LWC models for the cloud and drizzle, parameterized using Eqs. (8) and (20). The retrieval scheme is as follows. $\mathrm{LWC}_{\text {cld }}$ and $N_{\text {cld }}$ are first constructed to derive $Z_{\text {cld }}$. $Z_{\mathrm{dzl}}$ follows from the difference between the synthetic $Z$ and $Z_{\text {cld }}$. Based on the vertical extent of $Z_{\mathrm{dzl}}, \mathrm{LWC}_{\mathrm{dzl}}$ is constructed. The effective radius of drizzle is derived using Eq. (12) and the other properties follow from Eqs. (2)-(7).

The optimized total $Z$ (panel Ia), the attenuated backscatter (Ic), and $T_{\mathrm{B}}$ (Id) match the synthetic signals very well. The decomposition of the retrieved $Z$ into a cloud part and a drizzle part is shown in panel (Ib). The retrieved $Z_{\text {cld }}$ provides a good match to the truth. The true cloud LWC (Ie), effective radius (If), and extinction coefficient (Ig) are recovered with a high accuracy: the maximum difference between the truth and the retrieved values at any range gate amounts to no more than $5 \%$.

As for the drizzle, the retrieved $Z_{\mathrm{dzl}}$ (panel $\mathrm{Ib}$ ) is not a perfect match to the truth in the upper half of the cloud, where the lidar signal is weak. The drizzle properties are small in magnitude, especially in the upper part of the cloud, making their retrieval very sensitive to noise. Additionally, drizzle retrieval is based on excess $Z$, which means that any discrepancy between the retrieved and the true $Z_{\mathrm{cld}}$ has to be compensated by the retrieved $Z_{\mathrm{dzl}}$. Since $Z_{\mathrm{dzl}}$ is typically much lower than $Z_{\text {cld }}$ at the top part of the cloud, this compensation mechanism impacts the $Z_{\mathrm{dzl}}$ retrieval quite strongly. In some observational cases (see Sect. 4), as also reflected in this example, $Z_{\mathrm{dzl}}$ close to the cloud top can be very low, e.g., about $1 \%$ of the $Z_{\text {cld }}$, making it comparable to the noise or uncertainty level. In this sense, the droplet size threshold that we apply (Sect. 2.2) helps to distin- 


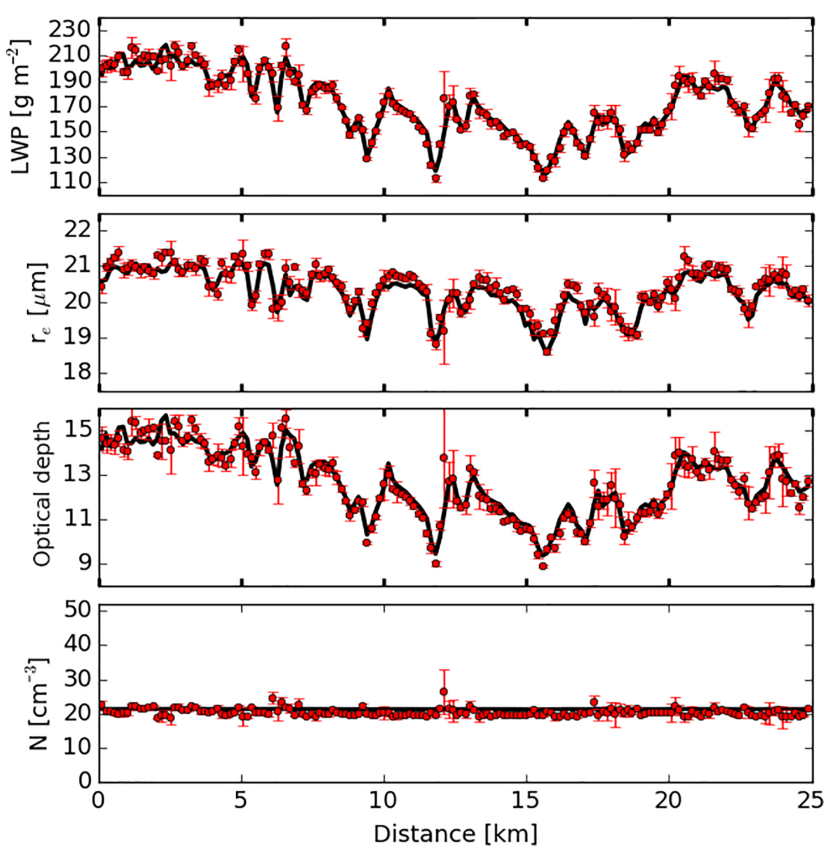

Figure 5. Microphysical and optical properties collapsed along the vertical axis: $N$ is column averaged; LWC and $\alpha$ are integrated into LWP and optical depth, respectively; and $r_{\mathrm{e}}$ are vertically averaged with the corresponding $\alpha$ as the weights. The black line represents the truth and the circles are the retrieved values. The error bars denote the random errors obtained from the Monte Carlo realizations.

guish weak drizzle signal from noise, but the retrieval accuracy would still be limited. Panels (Ii)-(Il) show the drizzle properties as derived from the knowledge of the drizzle LWC and $Z$. While the vertical shape is not exactly recovered, the retrieved values are within 1 order of magnitude from the truth.

- Case II

This is the case where isolated drizzle can be found below the cloud base, so here we can be more certain about the drizzle spatial extent. As in case I, the retrieval starts with building $\mathrm{LWC}_{\mathrm{cld}}$ and $N_{\mathrm{cld}}$ to derive $Z_{\mathrm{cld}} . Z_{\mathrm{dzl}}$ is computed from the difference between the synthetic $Z$ and $Z_{\text {cld }}$ above the cloud base; below the cloud base, $Z_{\mathrm{dzl}}$ is equal to the synthetic $Z$. Then, the profile of the drizzle effective radius is constructed using the two parameterizations given in Eqs. (13)-(14). The other drizzle properties follow from Eqs. (2)-(7).

The optimized total $Z$, the attenuated backscatter, and $T_{\mathrm{B}}$ are shown in panels (IIa), (IIc), and (IId). The decomposition of $Z$ (panel IIb) in the upper half of the cloud is plagued by the same problem as in case I. Just below the cloud top, the separation between the cloud and the drizzle contribution deviates from the truth, and this deviation is propagated to the retrieved properties (panels IIe-IIh for cloud and IIi-IIl for drizzle). The retrieved cloud LWC and cloud extinction coefficient are too large by $\sim 20-25 \%$ at the peak close to the cloud top. The vertical shape of the drizzle $r_{\mathrm{e}}$ follows an exponential function within the cloud. The retrieved effective radius here is about $6 \mu \mathrm{m}$ smaller than the truth. The smooth exponential profile of the drizzle $r_{\mathrm{e}}$ causes a dip in the $Z_{\mathrm{dzl}}$ value at $530 \mathrm{~m}$ to also appear in the $\alpha_{\mathrm{dzl}}$ and $N_{\mathrm{dzl}}$ profiles. This dip in drizzle $Z$ comes about because the cloud reflectivity between 500 and $600 \mathrm{~m}$ is not well recovered (the effect is most visible in panels IIe and IIg in Fig. 6). More specifically, the retrieved cloud $Z$ at these heights is slightly larger than the true ("observed") $Z$, resulting in $Z_{\text {excess }}=0$ (Eq. 19). The smoothing that is applied afterwards to produce $Z_{\mathrm{dzl}}$ replaces the zeros with nonzero values that are smaller than the neighboring range gates, creating the dip.

Below the cloud base, the drizzle $r_{\mathrm{e}}$ is restricted so that it does not decrease towards the drizzle base. Here we see that it is roughly constant with height. The retrieval scheme in this region relies on inferring the magnitude of the drizzle extinction coefficient from the lidar backscatter. The three control points (i.e., the drizzle extinction coefficients at three height levels) are retrieved with limited accuracies. The differences between the retrieval and the truth are on average about 38, 14, 46, and $52 \%$ of the true values for the drizzle LWC, $r_{\mathrm{e}}$, $\alpha$, and number concentration, respectively. Note that in this example, the small amount of drizzle extinction hardly changes the lidar backscatter below the cloud base. The drizzle extinction coefficient here amounts to about $5 \%$ of the molecular extinction. Other simulations were conducted where the amount of drizzle was varied. It was found that the drizzle retrieval accuracy below the cloud base increased as drizzle amounts increased. For example, when $\alpha_{\mathrm{dzl}}$ was increased by a factor of $\sim 20$, the retrieval accuracies improved: the drizzle LWC, $r_{\mathrm{e}}$, and $\alpha$ below the cloud were retrieved within $8 \%$, while the number concentration was within $25 \%$ from the truth (not shown).

In general, the cloud retrieval is more robust than the drizzle retrieval. Although the retrieved cloud properties for an individual cloud profile at a given height can be off by $\sim 25 \%$, the collective retrievals of a sample of profiles and column-averaged properties are expected to have better accuracies, as shown in Sect. 3.1. Drizzle retrieval is very sensitive to non-idealized cloud structure and also to the uncertainties of the observed signals. From the examples using idealized profiles of drizzle, it is shown that the retrieval error at a given range gate can be large (starting at $30 \%$ level) but, overall, profiles of the retrieved drizzle properties are reasonably close to the truth. 

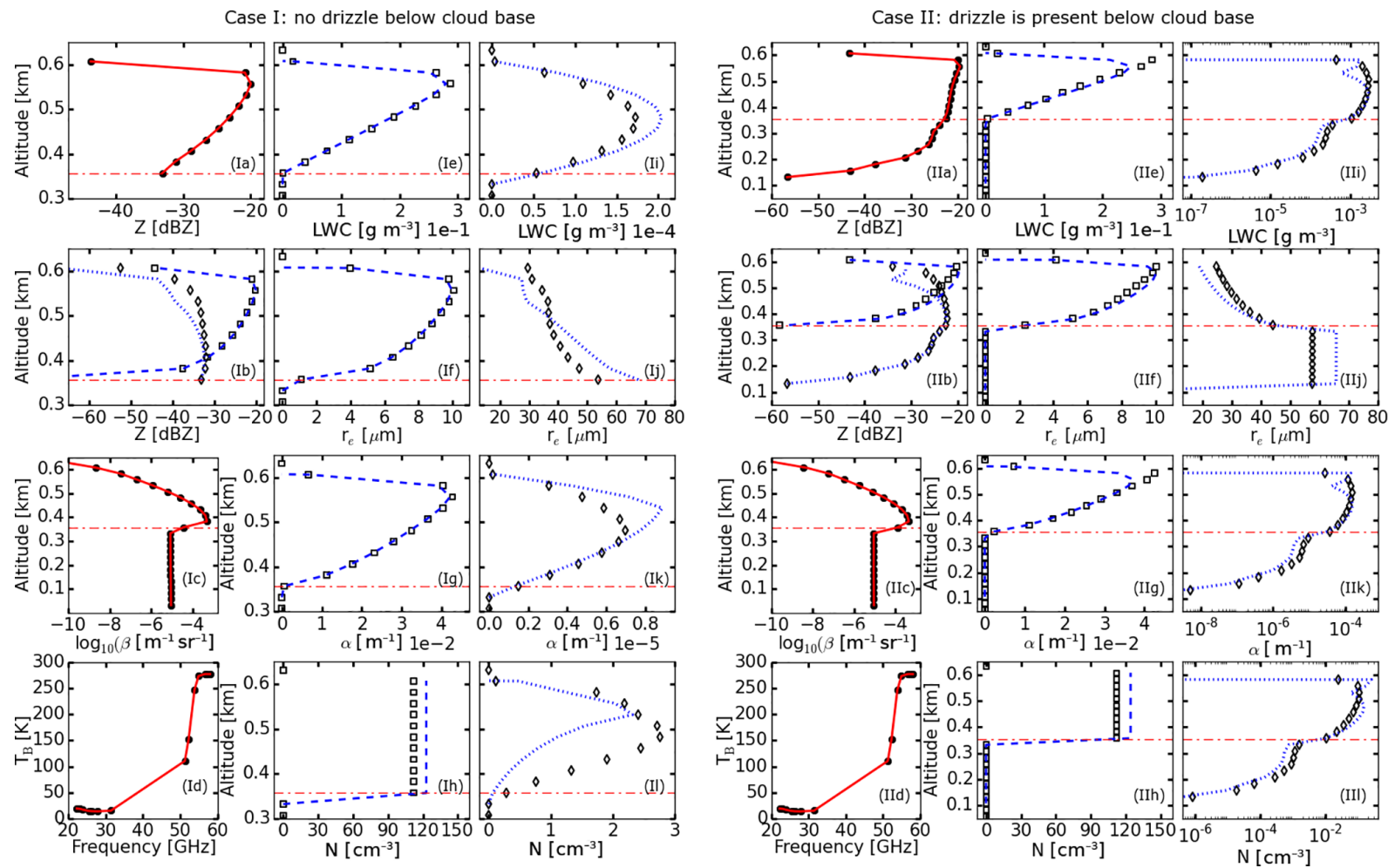

Figure 6. Examples of cloud and drizzle profiling for the two drizzle cases described in Sect. 2.2.2. Panels (Ia)-(II) show the results for case I and panels (IIa)-(III) for case II. The red dashed-dotted lines mark the cloud base height $z_{\mathrm{cb}, \text { opt }}$. Filled circles: synthetic signals; squares: the cloud truth; diamonds: drizzle truth; red solid lines: retrieved signals; blue dashed lines: retrieved cloud properties; blue dotted lines: retrieved drizzle properties.

\section{Application to ground-based observations}

The observational data were collected during the ACCEPT campaign that took place in October and November 2014 in Cabauw, the Netherlands (see Myagkov et al., 2016, and Pfitzenmaier et al., 2017, for more information about the measurement campaign). We use the data acquired from three co-located instruments:

- A zenith-pointing MIRA-35 radar.

It is a Ka-band cloud radar with Doppler capabilities. The signal was recorded with a spatial resolution of about $30 \mathrm{~m}$.

- A UV lidar (Leosphere ALS 450) operating at $355 \mathrm{~nm}$. The attenuated backscatter measurements are available every $30 \mathrm{~s}$ with a vertical resolution of $15 \mathrm{~m}$.

- A microwave radiometer HATPRO.

The brightness temperature was measured at 14 frequency channels: the first seven between 20 and $35 \mathrm{GHz}$ and the other seven between 50 and $60 \mathrm{GHz}$. The temporal resolution is $1 \mathrm{~s}$ with regular gaps due to automatic calibration periods.

For the inversion procedure, we use the calibrated radar reflectivity factor, as well as model forecast of temperature and humidity delivered in the Cloudnet categorization product (Illingworth et al., 2007). The calibrated reflectivity here is already corrected for gas attenuation and has the same temporal resolution as the lidar although their time stamps do not exactly coincide.

The retrieval is performed on a column-by-column basis with a time interval of $30 \mathrm{~s}$. For each 1-D column, a set of radar, lidar, and MWR data was created by first finding the lidar and radar profiles that are less than $15 \mathrm{~s}$ apart. The corresponding $T_{\mathrm{B}}$ profile was computed by averaging $T_{\mathrm{B}}$ measurements within $15 \mathrm{~s}$ of the average time stamp of the radar and lidar. The standard deviation of the mean was then adopted as the measurement error. Since the full overlap distance of the lidar is expected to be around $100-200 \mathrm{~m}$, column profiles with radar detections down to $<200 \mathrm{~m}$ were not retrieved. There are gaps in the observations where a complete dataset for the three instruments is not available, e.g., breaks in the MWR data stream during instrument calibration periods.

We selected two periods with a total time duration of approximately $4 \mathrm{~h}$ on 25 and 26 October when one layer of liquid water cloud was present. The scene includes clouds with clear precipitation events and also clouds without an obvious signature of drizzle below the cloud base, which is suitable for the dual retrieval mode (case I and case II). The cloud top 

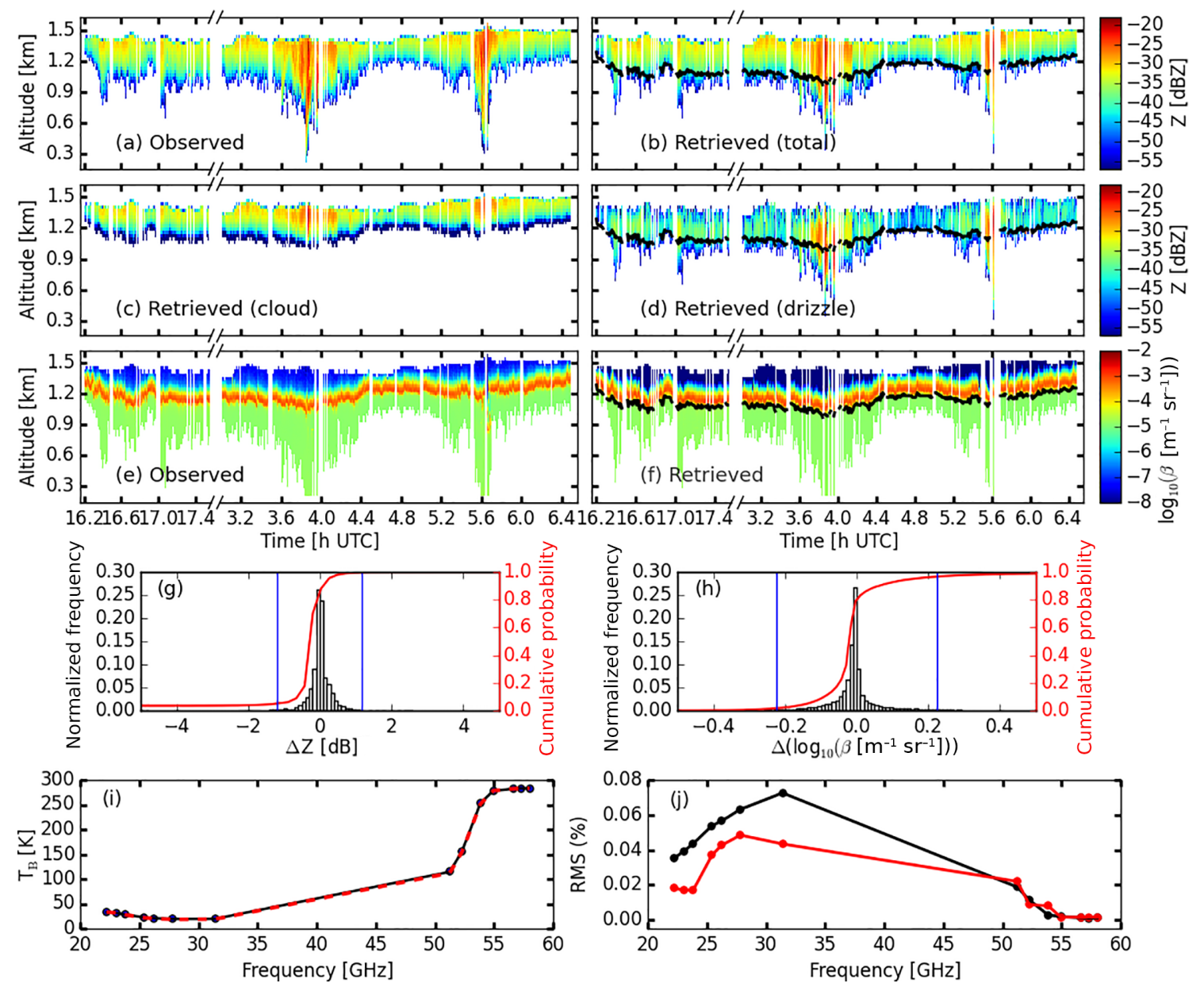

Figure 7. Measured and retrieved signals of the selected cases from the ACCEPT campaign. The breaks along the horizontal axes in (a)(f) mark the change of date from 25 to 26 October 2014. Panels (a)-(d) show the radar reflectivity as observed and as retrieved, along with the decomposition into drizzle and cloud reflectivities. The black line delineates the cloud base determined in the retrieval. Panels (e)-(f) display the observed and the retrieved attenuated lidar backscatter $\beta$. Panels (g) and (h): histograms of the differences between the observed and the retrieved (total) signals (observed minus retrieved). The red curves show the cumulative distributions. The spread of the distribution is indicated by the blue vertical lines that mark the interval within which $95 \%$ of the total occurrences are found. Panel (i) shows the brightness temperatures $T_{\mathrm{B}}$ averaged over distance at each frequency channel: the black circles and line show the observations while the dashed red line shows the retrieval. In (j) we plot the standard deviation of the observation mean divided by the mean itself (black) and the RMSD between the retrieval and the observation divided by the observation mean (red).

is located between 1400 and $1500 \mathrm{~m}$, with the cloud thickness varying between 200 and $400 \mathrm{~m}$. The cloud base height (as determined in the retrieval) fluctuates between 1050 and $1250 \mathrm{~m}$ during the two periods. Despite low reflectivity values, virga is observed below the cloud base for the majority of the time, with its maximum occurring on 26 October. The extent of the drizzle below the cloud is variable, with a depth of up to $600 \mathrm{~m}$.

The observed signals and their recovery in the retrieval are shown in Fig. 7 for each instrument. In general, the reflectivity within the cloud increases with height, indicating particle condensational growth. For the most part, the radar reflectivity is not higher than $-28 \mathrm{dBZ}$. On 26 October, at around 3.8 UTC (all times are in $24 \mathrm{~h}$ decimal format; e.g.,
17:00 UTC is represented by 17.0 UTC), $Z$ is maximum at $-12 \mathrm{dBZ}$. In the retrieval, cloud and drizzle contributions to the total reflectivities are separated and are shown in panels (c) and (d). Below the cloud base, $Z$ belongs only to the drizzle. Above the cloud base, the reflectivity of the cloud increases with height and peaks close to the cloud top. Conversely, drizzle reflectivity increases downwards from the top and reaches maximum in the cloud base region before decreasing again towards the drizzle base. It follows that within the cloud the cloud reflectivity dominates towards the cloud top while drizzle dominates near the bottom. In almost all profiles where no virga is visible below the cloud base, the retrieval algorithm finds drizzle to be present within the cloud, although with small reflectivities. This is usually caused by 
the significant excess reflectivity near the cloud base that cannot be attributed to the cloud component.

Panels (e) and (f) compare the observed and retrieved lidar attenuated backscatter. The fitting of the $\beta$ profile starts from an altitude between $200 \mathrm{~m}$ (from the ground) and the drizzle base and continues up to $200 \mathrm{~m}$ above the cloud base. Several lidar $\beta$ profiles show double backscatter peaks that we deem unsuitable for the algorithm, in which case the retrieval is not performed, resulting in white gaps in the time-height map. We found 25 such column profiles, corresponding to $5 \%$ of the available data. It can be seen that the drizzle below the cloud base remains largely transparent and undetected by the lidar. The histograms of the residual signals are similar to those obtained for the LES exercise (Fig. 3e and f): centered at zero and largely symmetric. Compared to Fig. 3e, the $\Delta Z$ distribution here is narrower because a part of the $Z$ residual is attributed to drizzle. The mean brightness temperature at each frequency channel is shown in Fig. 7i. The observations show little variation over time (less than $8 \%$ ). On average, the fit to the observed $T_{\mathrm{B}}$ is reasonably good with an RMSD of $5 \%$ or less, as indicated by the red line. The largest variation or difference is seen around $30 \mathrm{GHz}$, where the cloud contribution to the microwave extinction spectrum is significant.

The retrieved microphysical and optical properties for both cloud and drizzle are shown in Fig. 8. The cloud and drizzle LWC in panels (a) and (b) show a similar time-height distribution to the respective reflectivity field. Cloud LWC increases with height until the peak is reached close to the cloud top, while most water in drizzle is found at a lower altitude. The highest LWC is found at the time of maximum observed reflectivity. Drizzle water content is generally 2 orders of magnitude smaller than the cloud LWC. The averages of the $\mathrm{LWC}_{\mathrm{cld}}$ and $\mathrm{LWC}_{\mathrm{dzl}}$ maximum values are $1.9 \times 10^{-1}$ and $1.2 \times 10^{-3} \mathrm{~g} \mathrm{~m}^{-3}$, respectively. The temporal variations in the cloud and drizzle LWP are positively correlated in time, as was also found by Fielding et al. (2015).

The effective radius of the cloud droplets (panel c) is found to be well below the threshold values of $13 \mu \mathrm{m}$. As the cloud droplets grow via condensation, their size increases with height to a peak value of $5.1 \mu \mathrm{m}$ on average. Drizzle effective radius (panel $\mathrm{d}$ ), on the other hand, increases towards the cloud base as imposed by the parameterization. At the cloud base, the mean drizzle effective radius is found to be $\sim 22 \mu \mathrm{m}$. During the intense drizzle period between 3.8 and 4.0 UTC, it can be as high as $60 \mu \mathrm{m}$.

The extinction coefficients of the cloud and drizzle (panels e and f) are mainly determined from the observed lidar attenuated backscatter. The extinction coefficient of drizzle is smallest below the cloud base, in accordance with the relatively low observed $\beta$ in this region. It increases with height and peaks within the cloud but it is still orders of magnitude smaller than the cloud. The mean maximum $\alpha_{\text {cld }}$ and $\alpha_{\text {dzl }}$ values are 0.06 and $1.2 \times 10^{-4} \mathrm{~m}^{-3}$, respectively. The number concentration of the cloud (panel $\mathrm{g}$ ) droplets shows a somewhat high and rapid fluctuation. From our LES exercise (Sect. 3), we learned that setting $v_{\text {cld }}$ as a free parameter can indeed cause this, but we also expect the fluctuation to average out to a minimally biased mean value. The cloud number concentration averages to about $549 \mathrm{~cm}^{-3}$. The drizzle droplet concentration (panel h) has a mean of $\sim 0.06 \mathrm{~cm}^{-3}$, which is consistent with the in situ measurements of marine stratocumulus clouds in the MASE-II experiment (Lu et al., 2009).

In Fig. 9 we show the mean vertical profiles of the radar reflectivity and the derived microphysical properties of the cloud and drizzle. These profiles are constructed by averaging the retrieved profiles between 3.8 and 4.0 UTC when there is significant drizzle. In all but the lowermost parts of the cloud, drizzle makes a very small contribution to the total water content. Close to the cloud top, the effective radius of the drizzle droplets is found to be around $30 \mu \mathrm{m}$ for this particular time segment. At around the same height, the cloud droplets reach their maximum size of $\sim 6 \mu \mathrm{m}$, with the cloud liquid water content and reflectivity dominating over the drizzle. As the drizzle droplets grow exponentially towards the cloud base via coalescence, drizzle reflectivity increases, matching the cloud reflectivity about halfway through the cloud. Near the cloud base, drizzle reflectivity becomes dominant due to the much larger size of the drizzle droplets compared to the cloud droplets. Inside the cloud layers, this behavior of cloud and drizzle reflectivities is found to be quite typical over the observation period. From the cloud base towards the drizzle base, $Z_{\mathrm{dzl}}$ decreases monotonically and so does the $\mathrm{LWC}_{\mathrm{dzl}}$. Once drizzle drops escape the cloud, they are expected to evaporate below the cloud base and shrink as they fall through the air, in accordance with the gradient seen in the $r_{e}$,dzl profile. The jagged feature in the $r_{e, \text { dzl }}$ profile below the cloud base is an artifact of the profile averaging caused by the variable drizzle base height during the $12 \mathrm{~min}$ period.

Lastly, the number concentration of the drizzle is 3-4 orders of magnitude smaller than the cloud at all heights above the cloud base. Unlike the cloud number concentration, $N_{\mathrm{dzl}}$ shows variation in height. The highest density of drizzle is found approximately where its LWC is highest - that is, within the cloud. From this point towards the cloud base, the drizzle number density keeps decreasing as $r_{e, \text { dzl }}$ rises sharply, which could be due to the accretion of smaller drizzle droplets by the bigger ones to form even larger droplets. Below the cloud base, some droplets experience complete evaporation, depleting the number density of drizzle as it approaches the ground.

\section{Comparisons with other retrieval methods}

To assess the results presented in Sect. 4, we perform comparisons with three independent retrieval methods applied to the ACCEPT dataset within the same period. The three re- 

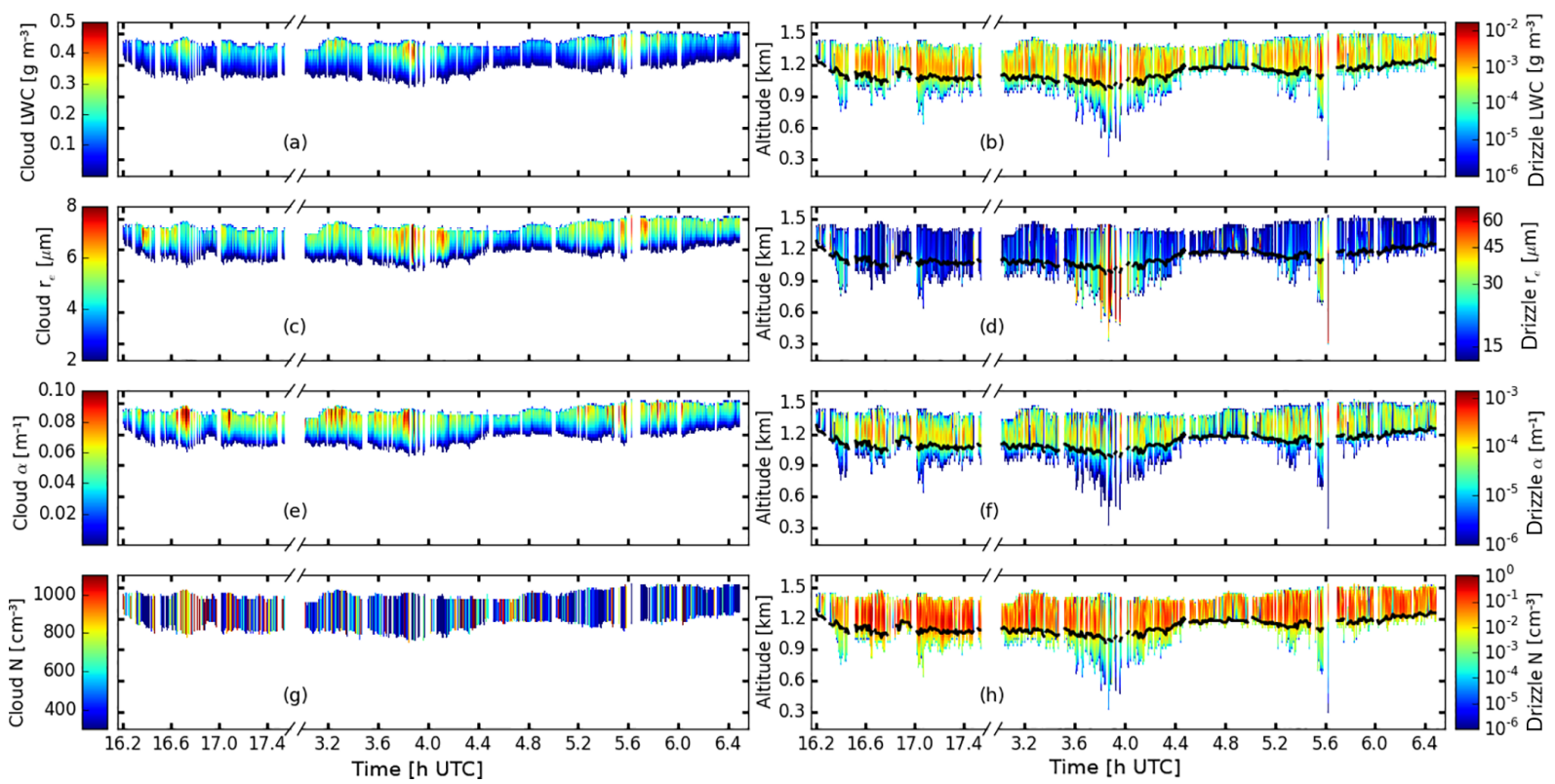

Figure 8. Optical and microphysical parameters of the cloud $(\mathbf{a}, \mathbf{c}, \mathbf{e}, \mathbf{g})$ and the drizzle $(\mathbf{b}, \mathbf{d}, \mathbf{f}, \mathbf{h})$ as obtained from the retrieval, as a function of time and height. From top to bottom: liquid water content, effective radius, extinction coefficient, and number concentration.

trieval techniques offer a tool to evaluate our retrieval below and above the cloud base separately. Below the cloud base, the drizzle comparison is made with the readily available results from the method of O'Connor et al. (2005) as part of the Cloudnet algorithm package. Above the cloud base, the cloud properties are retrieved using the depolarization lidar (DL) technique developed by Donovan et al. (2015). The amount of drizzle within the cloud is derived from radar Doppler spectra analysis, as described in Kollias et al. (2011a, b). In Appendix A, we present the application of this technique to the ACCEPT data, following the implementation in Luke and Kollias (2013); the comparison with our retrieval is discussed in Sect. 5.2.

\subsection{Cloud properties above the cloud base}

A depolarization-lidar-based method (Donovan et al., 2015) was applied to the lidar dataset used in our retrieval to derive the cloud properties. While our retrieval method utilizes only the total attenuated backscatter, the DL method exploits the parallel and perpendicular polarization components of the received signal to infer the cloud extinction coefficient and droplet size.

Figure 10 displays the time series of the cloud properties at a specific, arbitrary height (chosen to be $100 \mathrm{~m}$ ) above the cloud base, as derived from the DL (blue) and our (black, red) retrieval methods. The DL method determines the cloud base height that is then used as a height reference for the subsequent retrieval of the extinction coefficient and effective radius, from which the LWC and $N$ are then derived. The retrieval is performed with a temporal resolution of $180 \mathrm{~s}$.
For a fair comparison, we interpolate our retrieved profiles to $100 \mathrm{~m}$ above the cloud base as well. Since our retrieval has a higher temporal resolution ( $30 \mathrm{~s}$ ), we use our own cloud base height estimate $\left(z_{\mathrm{cb}}\right)$ as a reference. It is therefore imperative to first make sure that the cloud base height estimates from the two methods match. The bottom panel of Fig. 10 shows that this is indeed the case.

We average our results over the $180 \mathrm{~s}$ time interval to match the time stamps and the temporal resolution of the DL method. The time-averaged products (red line in Fig. 10) are then compared with the results of the DL method. The extinction coefficients retrieved by both methods are very similar; we find that the mean difference and the RMSD amount to -5 and $11 \%$, respectively. The fractional quantities here and in the following are produced using the mean of the DL results as a reference. The effective radii do not compare as well, with a mean difference of $-10 \%$ and an RMSD of $21 \%$. Our effective radius is highest at around 3.88 UTC, coinciding with a high radar reflectivity situated in the middle of an intense drizzle episode. This is not seen in the DL retrieval. The difference in $r_{\mathrm{e}}$ is mostly visible between 17.0 and 17.4 UTC as well as between 4.8 and 5.4 UTC. The mismatch does not necessarily correlate with the drizzle quantity and can amount to $1.5 \mu \mathrm{m}$ but is still within the expected DL uncertainty range.

Our retrieved LWC is on average lower by about $15 \%$ with an RMSD of $26 \%$. The DL number concentration is derived assuming that the DSD follows a single-mode gamma distribution (Eq. 1) with a shape parameter $v=6$. Uncertainties of $N$ are propagated from the errors of $r_{\mathrm{e}}$ and $\alpha$, taking into account a range of shape parameter values between 4 and 10 

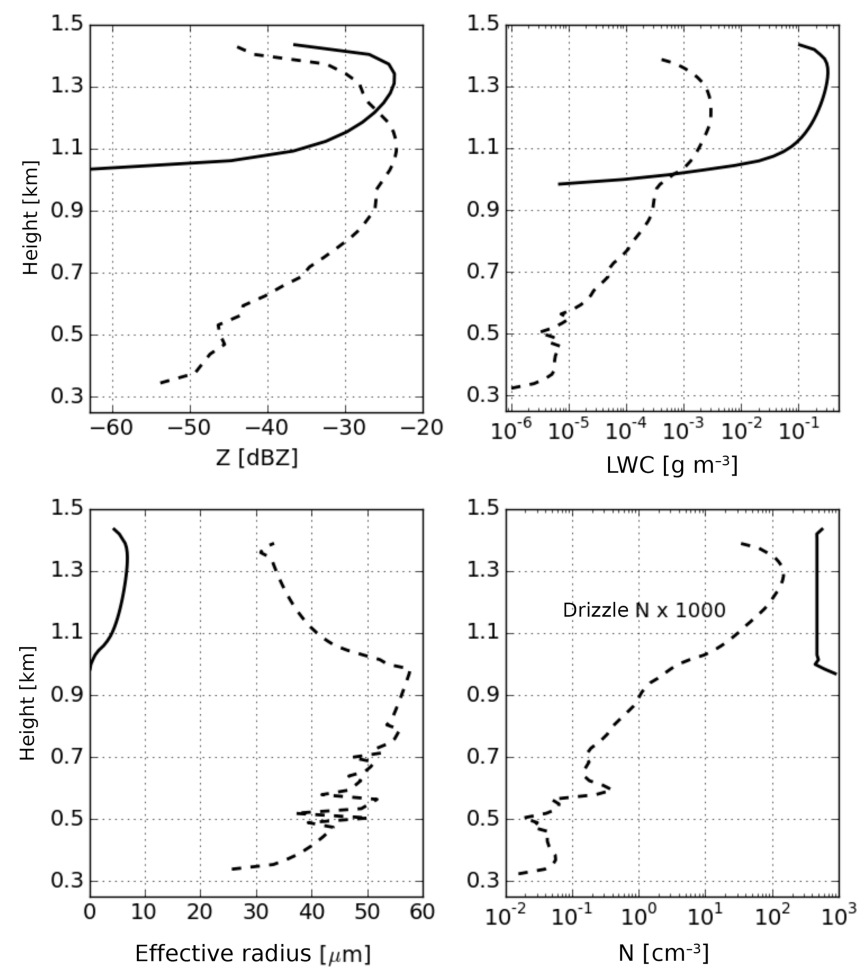

Figure 9. Mean vertical profiles of the cloud (solid lines) and drizzle (dashed lines) properties from the observation on 26 October 2014 between 3.8 and 4.0 UTC. Clockwise from the top left: radar reflectivity factor in $\mathrm{dBZ}$, liquid water content in $\mathrm{g} \mathrm{m}^{-3}$, number density per $\mathrm{cm}^{3}$ and effective radius in $\mu \mathrm{m}$. Note that the number density of the drizzle has been multiplied by a factor of 1000 for illustration purposes.

which is typical for liquid water clouds. Our number concentration fluctuates rather strongly with time, contributing to the large RMSD (45\%). The shape parameter in the retrieval procedure is set as a free parameter to minimize biases and, as discussed in Sect. 3, this can lead to a strong fluctuation in the number density. In comparison to the DL method, our mean $N$ is higher by about $15 \%$. Given that the DL uncertainties of $\alpha$ are between 15 and $20 \%$ and that the large fraction of the DL-retrieved $r_{\mathrm{e}}, \mathrm{LWC}$, and $N$ have $\sim 50 \%$ errors, the differences between the two methods are well within the range of uncertainties.

\subsection{Drizzle reflectivity above the cloud base}

The qualitative comparison between our retrieval results and the Doppler retrieval results is shown in Fig. 11 for the drizzle (a), cloud (b), and the total (c) reflectivities. Each circle represents a time-height pixel for which both methods are applicable; the number of time-height pixels that can be retrieved using only one of the methods, and hence are not used in the comparison, is less than $8 \%$. In general, the distribution of the circles is consistent with the one-to-one line. The spread is higher for lower $Z$, indicating the higher fraction of noise for the low- $Z$ retrieval. The larger values of our (retrieved) total $Z$ in (Fig. 11c) compared to the Doppler counterpart can be attributed to the absence of attenuation correction in the Doppler spectra. On average, there is a difference of $0.9 \mathrm{~dB}$ between the two sets of total $Z$. This difference has very little effect on the trend that we see in the scatter plots (Fig. 11a) and (Fig. 11b), in which the circles are color coded according to their relative heights within the cloud. We divide the cloud into three horizontal parts: (i) one quarter into the cloud (cloud base region, red), (ii) the top quarter (cloud top region, green), and (iii) the middle region in between (blue). The insets show the same scatter plot without the blue points to highlight the division between the cloud base and cloud top regions.

The correlation between our $Z$ and Doppler $Z$ varies across altitudes within the cloud. Close to the cloud base there is a clear tendency for our retrieved drizzle reflectivities to be larger than those from the Doppler analysis. The cluster of red circles in (Fig. 11a) is almost exclusively located to the left of the one-to-one line. Consequently, our cloud reflectivities become smaller than the Doppler $Z_{\text {cld }}$, as seen in (Fig. 11b). The primary cause for this stems from the assumptions used in both methods. In our retrieval, the cloud LWC, effective radius, and thus reflectivity near the cloud base are assumed to decrease downwards until they reach zero at the cloud base. This way, drizzle can gradually maximize its share of the total reflectivity, guaranteeing the continuity of the drizzle reflectivity when crossing the cloud base. Such a restriction in the vertical profile is not in place for the Doppler retrieval, and the applicability of the method is limited to situations where drizzle is not dominant.

It should be noted that the accuracy of the Doppler retrieval is crucially determined by the shape of the spectra. The choice of the time interval during which the individual spectra are averaged is known to play a role in determining the shape of the composite spectra (Giangrande et al., 2001). Here we fix the time window to $30 \mathrm{~s}$ to allow for a one-toone comparison with our retrieval results. High turbulence is shown to cause an underestimation of drizzle reflectivity, as derived through spectral decomposition, by up to $10-15 \mathrm{~dB}$ (Luke and Kollias, 2013). This means that, even if drizzle indeed dominates the cloud, turbulence can smear the spectrum in such a way that it appears cloud dominated. A compensation for this effect is thus critical in determining the correct drizzle fraction. In the scatter plots above, the presented reflectivity values have been turbulence corrected according to Luke and Kollias (2013), who estimate the correction factor as a function of spectral broadening, determined from their extensive dataset of marine stratocumulus clouds. Ideally, turbulence-corrected drizzle reflectivities just below and just above the cloud base should have similar values, which was indeed the case in Luke and Kollias (2013). After applying their procedure to the ACCEPT dataset, however, we find that the drizzle reflectivities above the cloud base are lower by several $\mathrm{dB}$ (the mean is $4 \mathrm{~dB}$ lower) than those just below, 

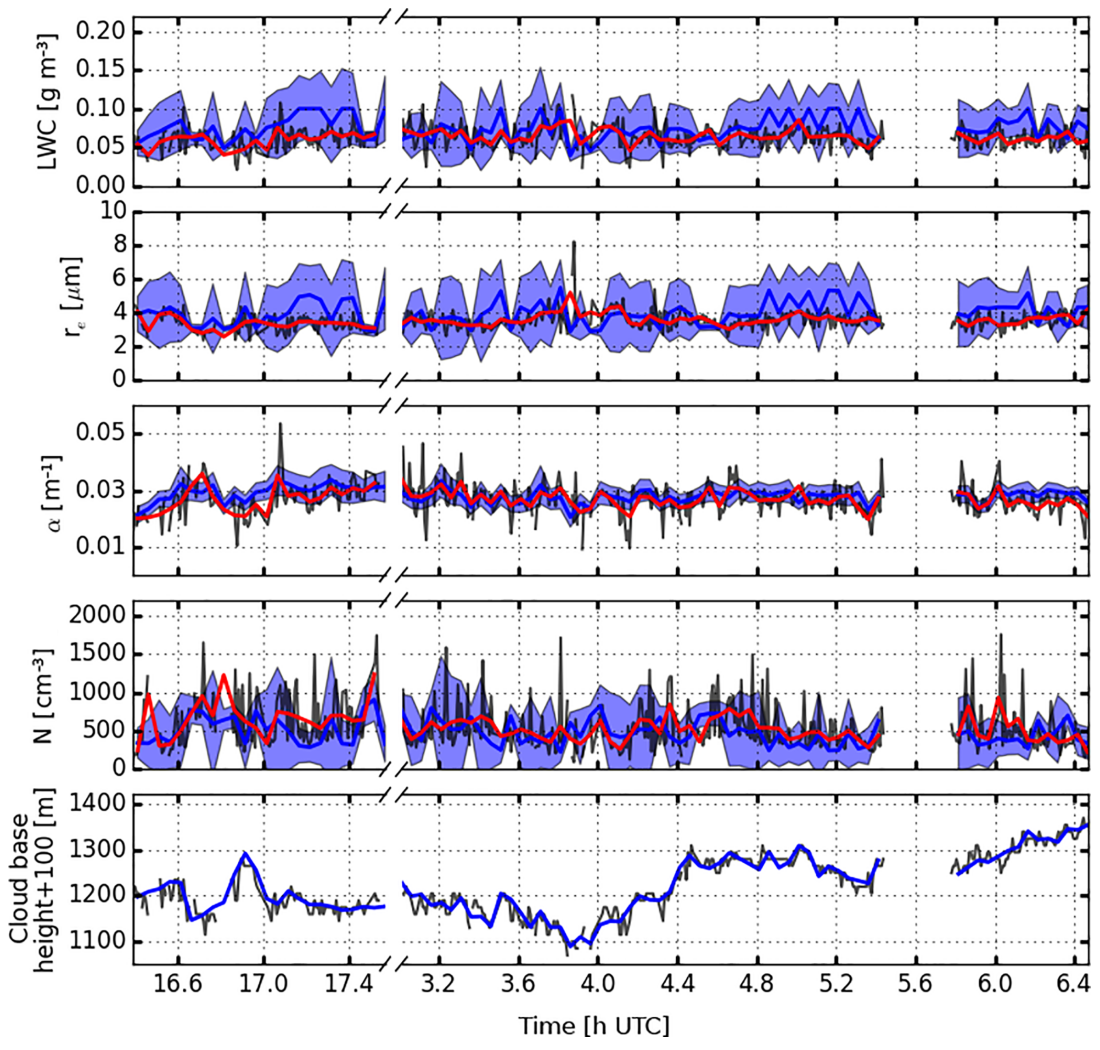

Figure 10. Time series of the optical and microphysical properties of the cloud at $100 \mathrm{~m}$ above the cloud base. The break along the horizontal axis marks the change of day from 25 to 26 October. The data around 5.6 UTC are deemed unsuitable for retrieval using the depolarization lidar method. The cloud properties and their respective uncertainties derived using the depolarization method are shown by the blue lines and the shaded area. The results of our retrieval are represented by the black lines (temporal resolution: 30 s) and red lines (time averaged to match the time stamps and the $180 \mathrm{~s}$ resolution of the depolarization results). Top to bottom: liquid water content, effective radius, extinction coefficient, number concentration, and the altitude at which the cloud properties are evaluated.
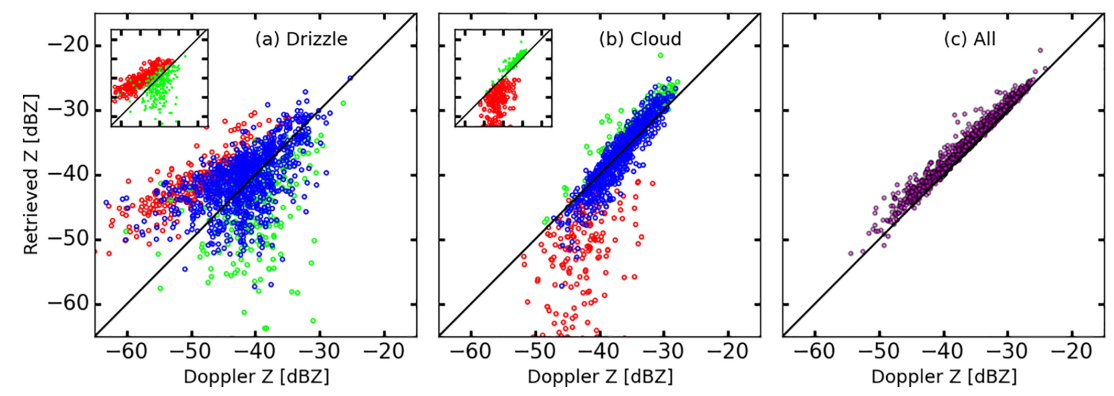

Figure 11. Radar reflectivities from our retrieval ("retrieved $Z$ ") plotted against those derived from the Doppler spectral decomposition ("Doppler Z"). Drizzle, cloud, and total reflectivities are shown separately in (a), (b), and (c), respectively. The points are color coded according to their location in the cloud. Red points are from the cloud base area, blue points are from the middle of the cloud, and green points are from the cloud top region (see the text for details). The insets in (a) and (b) show the same plot with the blue points excluded. The diagonal line is the one-to-one line.

as shown in Fig. 12. This could be the result of the uncertainties in the estimate of the correction factor or an artifact of the spectrum averaging process. We do not attempt to formulate the correction factor necessary to eliminate this reflectivity gap due to the insufficient amount of data needed to provide a statistically significant estimate.
Towards the cloud top there is a mild trend in which our retrieved drizzle reflectivity is smaller than that derived from the spectra, thus opposite to what is seen in the cloud base region. In our retrieval, the drizzle reflectivity is derived from the excess reflectivity that is not claimed by the cloud component and it typically increases towards the cloud base. Such 


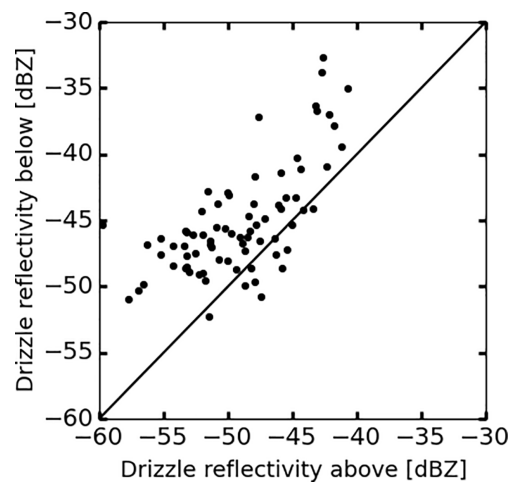

Figure 12. Comparison between the drizzle reflectivities at one range gate above and one range gate below the cloud base. The reflectivities above the cloud base are obtained from the Doppler spectral decomposition. The ones below the cloud base are simply the observed reflectivities. The diagonal line marks the one-to-one correspondence.

a pattern is not enforced and is also hardly visible in the Doppler retrieval, leading to discrepancies in the results.

The best agreement between the two methods is found for the middle part of the clouds. If a shift of a few $\mathrm{dB}$ were introduced to $Z_{\mathrm{dzl}}$ above the cloud base to correct for the reflectivity gap indicated in Fig. 12, then the agreement would improve in the cloud base and mid-cloud regions, but would worsen in the cloud top region. Overall, the correlation coefficient assessed by including all points is 0.38 for drizzle and 0.75 for the cloud. When we examine only the midcloud reflectivity distribution, the correlation coefficient improves to 0.54 and 0.87 for drizzle and cloud, respectively. The cloud boundaries are problematic areas for the comparisons due to their transitional nature, lower reflectivities, and therefore high uncertainties. However, despite the distinct retrieval procedures, different sources of information, and different assumptions, the two methods show reasonable agreement in quantifying the amount of drizzle within the cloud.

\subsection{Drizzle LWP below the cloud base}

Drizzle parameters below the cloud base are retrieved as one of the level 2a Cloudnet products using the algorithm introduced in O'Connor et al. (2005). The retrieval makes use of lidar backscatter and the first three moments of the Doppler radar spectra. The radar data come from MIRA radar, which is the same radar used for our retrieval. The lidar backscatter information is obtained from an independent observation with a different instrument, i.e., a CHM15X ceilometer.

The drizzle property that we can directly compare is the liquid water path of the drizzle below the cloud base, displayed in Fig. 13. The graph demonstrates a strong correlation between the two sets of LWP values, spanning a few orders of magnitude. The points align well with the one-toone line and the correlation coefficient is found to be 0.99 .

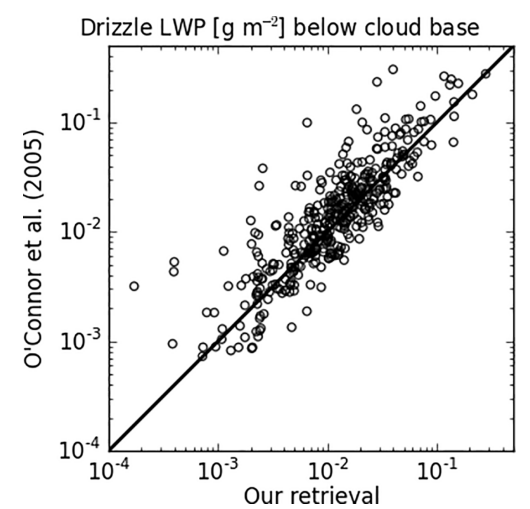

Figure 13. Comparison with the drizzle liquid water path below the cloud base as retrieved using the technique of O'Connor et al. (2005), available as one of the Cloudnet products. The diagonal line marks the one-to-one correspondence.

The mean values of $\log _{10}(\mathrm{LWP})$ are -2.15 (ours) and -1.87 (O'Connor et al., 2005, method) with an RMSD of 0.29.

\section{Summary}

We developed a method to simultaneously profile cloud and drizzle properties by exploiting the synergy of ground-based radar, lidar, and microwave radiometer measurements. This method has the advantage that the presence (or absence) of drizzle is inferred from the best fit to the data, rather than being imposed prior to the retrieval. The lidar forward model simulates the attenuated backscatter not only below the cloud base but also a few hundred meters into the cloud, taking multiple scattering into account. The cloud and drizzle components are distinguished using a droplet size threshold of $13 \mu \mathrm{m}-\mathrm{a}$ choice that is empirically motivated by the results of numerous observational and numerical studies. The combined droplet size distribution of cloud and drizzle follows a bimodal gamma distribution function. To aid the retrieval and to ensure some level of smoothness, the general shape of the cloud and drizzle vertical profiles is parameterized based on empirical findings in the literature. The retrieval products include a full set of microphysical parameters (LWC, droplet effective radius, and number density) and the optical extinction coefficient for the cloud and drizzle components.

The cloud retrieval was tested using synthetic signals generated from LES output. The vertical (along the height) and horizontal (along the time axis) variations in the true microphysical properties were largely reproduced, thereby verifying forward models and the cloud LWC model. On average, the column-averaged effective radius and the columnintegrated quantities (LWP and optical depth) were retrieved within $1 \%$ of the mean truth, while the number density was retrieved within $5 \%$. This indicates that, in the absence of radar calibration error, the retrieval method can potentially achieve a high accuracy. From this LES exercise, it appears 
that, when the radar calibration is inaccurate, assuming a single (incorrect) value of the shape parameter $v$ in the retrieval can introduce a significant bias. When $v$ was set as a free parameter in the fitting, temporal noise increased but the mean bias in the retrieved cloud properties decreased. A radar systematic error of $3 \mathrm{~dB}$ led to a mean bias of $\sim 15 \%$ in LWP, $r_{\mathrm{e}}$, and $N$, and this value increased when $v$ was fixed to the true value. Examples of the full cloud and drizzle retrieval using synthetic signals were given for the two drizzle cases implemented in the algorithm. The reflectivity decomposition into the cloud and drizzle components was most problematic in the upper half of the cloud. The retrieval of the cloud component appeared to be more robust than the drizzle.

The retrieval algorithm was applied to a dataset collected during the ACCEPT campaign in Cabauw, the Netherlands. Single layer liquid water clouds with a varying amount of virga were selected. The clouds were between 200 and $400 \mathrm{~m}$ thick with an LWP that varied mostly between 10 and $100 \mathrm{~g} \mathrm{~m}^{-2}$. The cloud and drizzle LWPs were found to be positively correlated, with the drizzle LWP being about 2 orders of magnitude smaller. The effective radius of the cloud droplets was found to be less than $5 \mu \mathrm{m}$ on average, which is far lower than the threshold value of $13 \mu \mathrm{m}$. The mean drizzle effective radius at the cloud base where it is expected to be the largest amounted to $\sim 22 \mu \mathrm{m}$ but increased to as high as $\sim 60 \mu \mathrm{m}$ in intense drizzle periods. The cloud number concentration averaged to about $549 \mathrm{~cm}^{-3}$, which is around 4 orders of magnitude larger than the drizzle number density. Such a ratio of cloud to drizzle number density is comparable to what has been measured for marine stratocumulus clouds in the MASE-II experiment (Lu et al., 2009).
Different elements of the ACCEPT retrieval products were assessed through comparisons with the results of three independent retrieval methods. The first method relied on the lidar depolarization signal to retrieve cloud properties at $100 \mathrm{~m}$ above the cloud base. The second one used radar Doppler spectra to quantify drizzle reflectivity within the cloud boundaries. Lastly, the third technique derived the drizzle LWP below the cloud base using information from both radar and lidar. Overall, the cloud and drizzle properties retrieved using the three retrieval methods and the method described in this paper show a high degree of consistency within the expected uncertainties. Considering the different approaches and the limitation of each retrieval technique, we find the agreement in the results encouraging.

In closing, the application examples of the retrieval algorithm presented here show promising results. The application to datasets with larger size and variety is necessary to establish and improve the validity of the method. Retrieval evaluations using radiances measured from space or on the ground as well as comparison with measurements of cloud condensation nuclei concentration could be part of the future development. From the computational point of view, there is room for optimization that would lead to a faster implementation of the algorithm and make it more suitable for a large-scale application (currently, it takes roughly $2.5 \mathrm{~h}$ for a dual core, $3 \mathrm{GHz}$ i7 MacBook Pro with $16 \mathrm{~GB}$ of RAM to process the $1.2 \mathrm{~h}$ ACCEPT data on 25 October 2014 shown in Figs. 7 and 8).

Data availability. The data used in this article are available upon request from the authors. 


\section{Appendix A: Drizzle retrieval using radar Doppler spectra}

Kollias et al. (2011a, b) introduced a method to improve drizzle retrieval within stratiform clouds by analyzing the higherorder moments of the radar Doppler spectrum. Luke and Kollias (2013) implemented this method and showed that it works successfully for almost $50 \%$ of the spectra close to the cloud top, decreasing to about $15 \%$ towards the cloud base. Here we apply the procedure in Luke and Kollias (2013) to the ACCEPT campaign dataset from the chosen time period in Sect. 4. The aim is to compare the drizzle reflectivity as derived from this spectral analysis with $Z_{\mathrm{dzl}}$ retrieved using the technique presented in this paper.

The Doppler spectra were acquired using the same cloud radar, so instrument calibration is not an issue. The spectra were recorded every second with a velocity resolution of $0.0825 \mathrm{~m} \mathrm{~s}^{-1}$ and a vertical resolution of $30 \mathrm{~m}$. For comparison purposes, we used the time-height coordinate in Fig. 7 such that one composite spectrum corresponds to one timeheight pixel. This means applying a running window of $30 \mathrm{~s}$ at a particular height above the cloud base. The spectra were shifted such that their spectral peaks coincided and were then averaged per velocity bin. The average velocity of the individual peaks was assigned to be the velocity location of the composite spectrum peak. From each composite spectrum, we computed the skewness as an indicator of drizzle presence. Negative skewness suggests that drizzle is the dominant component of the spectrum. Since the spectral decomposition technique is valid only for spectra with positive skewness, we continued to process only the composite spectra having skewness equal to or larger than 0.1 . Here, positive velocities correspond to downward motions (approaching the zenith-pointing radar).

Each spectrum was decomposed by assuming that the portion to the left of the maximum power was entirely due to the cloud - such that it represented the left half of cloud spectrum - and that the drizzle contribution remained to the right side of the spectral peak. The full cloud spectrum was constructed by assuming that the right half followed a Gaussian shape with a dispersion estimated from the known left portion. Having constructed a complete cloud spectrum, the drizzle spectrum was then produced from the difference between the cloud and the composite spectra.

With the cloud and drizzle spectra at hand, the reflectivity of each can be simply calculated from the zeroth moment. At this stage, we computed the correction factor to compensate for the turbulence as a function of the spectral broadening $\sigma_{t}$ (Fig. A2 in Luke and Kollias, 2013). For spectra with $\sigma_{t}$ larger than $0.1 \mathrm{~m} \mathrm{~s}^{-1}$, this correction was applied to the drizzle reflectivity. To preserve the total power, the cloud reflectivity was corrected (reduced) by the same amount.
Figure A1 presents the results of the procedure above. Panel (a) shows the observed (total) reflectivity computed from the zeroth moment of the composite spectra. This is comparable to the reflectivities shown in Fig. 7. Note that the reflectivities from the spectra are not corrected for gas attenuation, while those from the Cloudnet product are. The cloud base location is marked by the black line as determined from the lidar attenuated backscatter profile. The skewness displayed in panel (b) is strongly negative around 4 and 5.6 UTC, coinciding with high reflectivities and a high amount of drizzle below the cloud base. Spectral decomposition is not performed for pixels with skewness less than 0.1 and these show up as white gaps in panels (d)-(h).

The mean Doppler velocity shown in panel (c) demonstrates primarily updraft motion above the cloud base with a few downdraft streaks, most notably around 17.2 UTC. The mean velocity below the cloud base is strikingly higher than above the cloud base. This is indicative of falling drizzle drops that evaporate, cool the air, and cause downdraft motions. The reflectivity-weighted mean velocity for drizzle in panel (d) includes air motion to allow for a consistent comparison between velocities below and above the cloud base. Below the cloud base there is no retrieval so the velocity fields in panels (c) and (d) are identical. The cloud reflectivity shows similarities to the observed one, suggesting that the cloud is the dominant component. In panel (f), the drizzle reflectivities below the cloud base are identical to the observed reflectivities in (a). Visually, there is a continuous transition between the reflectivities above and below the cloud base. In Fig. 12, we show that there is a mean difference of $4 \mathrm{~dB}$ between the drizzle reflectivities immediately above or below the cloud base (see Sect. 5.2). Finally, the air motion in panel $(\mathrm{g})$ is determined from the average velocity of the spectral peaks within the $30 \mathrm{~s}$ time interval during the construction of composite spectra, and the width of the composite spectra is presented in panel (h). 

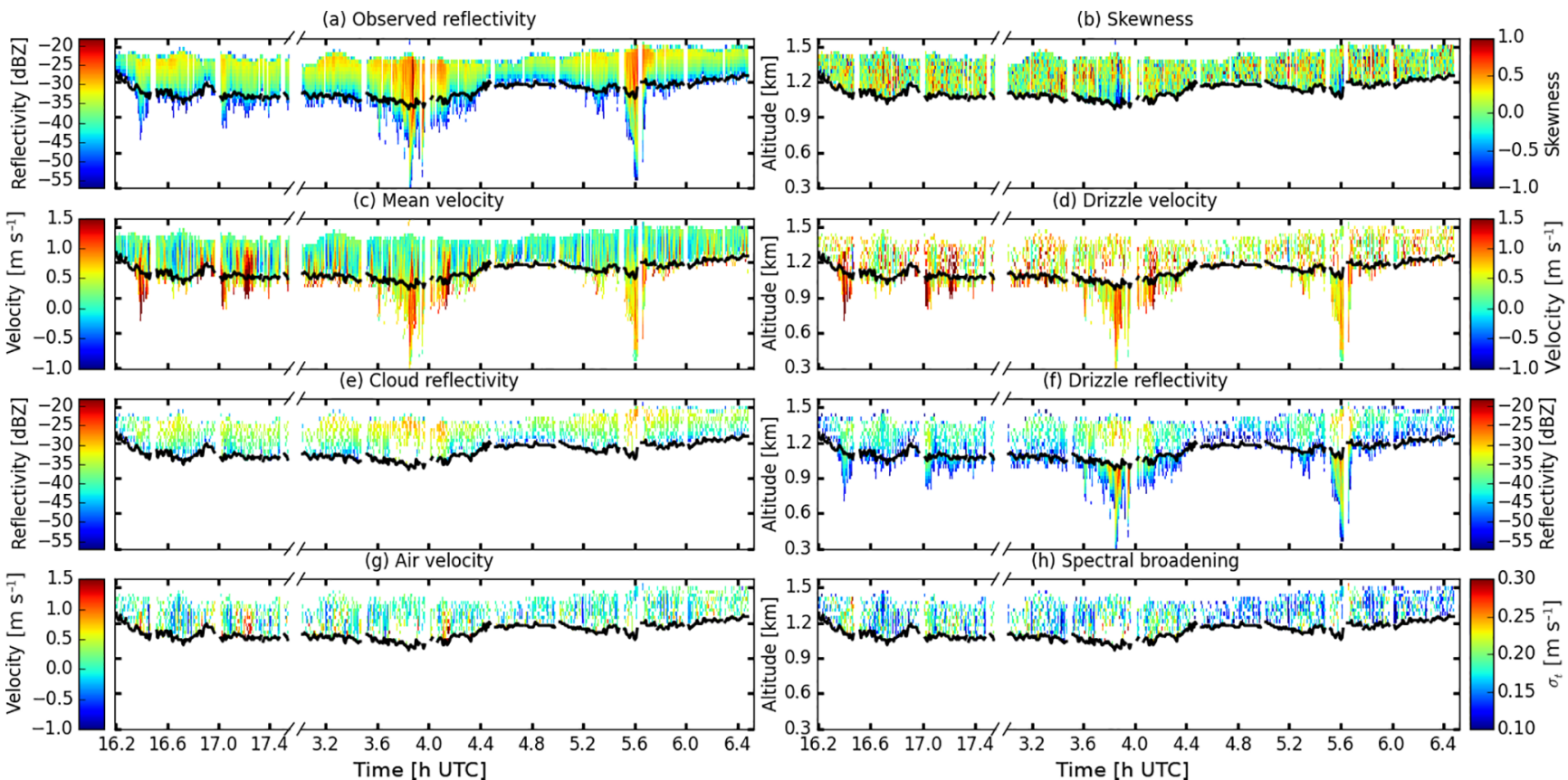

Figure A1. Results of the retrieval using Doppler spectra on 25 and 26 October 2014: (a) zeroth moment of the Doppler composite spectra; (b) skewness of the composite spectra above the cloud base; (c)-(d) first moments of the composite spectra and the drizzle spectra; (e)(f) zeroth moments of the cloud and the drizzle spectra, respectively, as obtained from the spectral decomposition; (g) air velocity; and (h) spectral broadening due to turbulence. The breaks along the time axis mark the change of day. The black line delineates the cloud base. 
Competing interests. The authors declare that they have no conflict of interest.

Acknowledgements. This research is supported by the Netherlands Organisation for Scientific Research (NWO) through the User Support Programme Space Research project ALW-GO/13-20. The ACCEPT campaign was partly funded by the European Union Seventh Framework Programme (FP7/2007-2013) under grant agreement no. 262254 and was carried out in cooperation with the Leibniz Institute for Tropospheric Research (TROPOS), LMU Munich, and METEK GmbH. The authors thank Lukas Pfitzenmaier (TU Delft) for his assistance with the ACCEPT dataset as well as Alexander Myagkov and Patric Seifert (TROPOS) for providing the clean radar Doppler spectra. We are grateful to Ulrich Löhnert for the provision of the original microwave radiative transfer code upon which the microwave forward model used in this work was based and to Christine Unal for the fruitful scientific discussions. We also acknowledge the suggestions from the two anonymous reviewers and the editor that improved the manuscript.

Edited by: Patrick Eriksson

Reviewed by: two anonymous referees

\section{References}

Albrecht, B. A.: Aerosols, Cloud Microphysics, and Fractional Cloudiness, Science, 245, 1227-1230, https://doi.org/10.1126/science.245.4923.1227, 1989.

Austin, R. T. and Stephens, G. L.: Retrieval of stratus cloud microphysical parameters using millimeter-wave radar and visible optical depth in preparation for CloudSat: 1. Algorithm formulation, J. Geophys. Res., 106, 28233-28242, https://doi.org/10.1029/2000JD000293, 2001.

Baedi, R., Boers, R., and Russchenberg, H.: Detection of Boundary Layer Water Clouds by Spaceborne Cloud Radar, J. Atmos. Ocean. Tech., 19, 1915-1927, https://doi.org/10.1175/15200426(2002)019<1915:DOBLWC>2.0.CO;2, 2002.

Benmoshe, N., Pinsky, M., Pokrovsky, A., and Khain, A.: Turbulent effects on the microphysics and initiation of warm rain in deep convective clouds: 2 -D simulations by a spectral mixedphase microphysics cloud model, J. Geophys. Res.-Atmos., 117, D06220, https://doi.org/10.1029/2011JD016603, 2012.

Boers, R., Jensen, J. B., and Krummel, P. B.: Microphysical and short-wave radiative structure of stratocumulus clouds over the Southern Ocean: Summer results and seasonal differences, Q. J. Roy. Meteor. Soc., 124, 151-168, https://doi.org/10.1002/qj.49712454507, 1998.

Boers, R., Acarreta, J. R., and Gras, J. L.: Satellite monitoring of the first indirect aerosol effect: Retrieval of the droplet concentration of water clouds, J. Geophys. Res.-Atmos., 111, D22208, https://doi.org/10.1029/2005JD006838, 2006.

Brandau, C. L., Russchenberg, H. W. J., and Knap, W. H.: Evaluation of ground-based remotely sensed liquid water cloud properties using shortwave radiation measurements, Atmos. Res., 96, 366-377, https://doi.org/10.1016/j.atmosres.2010.01.009, 2010.

de Roode, S. R. and Los, A.: The effect of temperature and humidity fluctuations on the liquid water path of non-precipitating closed- cell stratocumulus clouds, Q. J. Roy. Meteor. Soc., 134, 403-416, https://doi.org/10.1002/qj.222, 2008.

Donovan, D. P. and van Lammeren, A. C. A. P.: Cloud effective particle size and water content profile retrievals using combined lidar and radar observations, 1, Theory and examples, J. Geophys. Res.-Atmos., 106, 27425-27448, https://doi.org/10.1029/2001JD900243, 2001.

Donovan, D. P., Klein Baltink, H., Henzing, J. S., de Roode, S. R., and Siebesma, A. P.: A depolarisation lidar-based method for the determination of liquid-cloud microphysical properties, Atmos. Meas. Tech., 8, 237-266, https://doi.org/10.5194/amt-8237-2015, 2015.

Duynkerke, G. P., de Roode, R. S., van Zanten Margreet, C., Calvo, J., Cuxart, J., Cheinet, S., Chlond, A., Grenier, H., Jonker, P. J., Köhler, M., Lenderink, G., Lewellen, D., Lappen, C.-L., Lock, P. A., Moeng, C.-H., Müller, F., Olmeda, D., Piriou, J.-M., Sánchez, E., and Sednev, I.: Observations and numerical simulations of the diurnal cycle of the EUROCS stratocumulus case, Q. J. Roy. Meteor. Soc., 130, 3269-3296, https://doi.org/10.1256/qj.03.139, 2004.

Eloranta, E. W.: Practical Model for the Calculation of Multiply Scattered Lidar Returns, Appl. Opt., 37, 2464-2472, https://doi.org/10.1364/AO.37.002464, 1998.

Feingold, G., Boers, R., Stevens, B., and Cotton, W. R.: A modeling study of the effect of drizzle on cloud optical depth and susceptibility, J. Geophys. Res., 102, 13527-13534, https://doi.org/10.1029/97JD00963, 1997.

Fielding, M. D., Chiu, J. C., Hogan, R. J., Feingold, G., Eloranta, E., O'Connor, E. J., and Cadeddu, M. P.: Joint retrievals of cloud and drizzle in marine boundary layer clouds using ground-based radar, lidar and zenith radiances, Atmos. Meas. Tech., 8, 2663 2683, https://doi.org/10.5194/amt-8-2663-2015, 2015.

Fox, N. I. and Illingworth, A. J.: The Retrieval of Stratocumulus Cloud Properties by Ground-Based Cloud Radar, J. Appl. Meteorol., 36, 485-492, https://doi.org/10.1175/15200450(1997)036<0485:TROSCP>2.0.CO;2, 1997.

Freud, E. and Rosenfeld, D.: Linear relation between convective cloud drop number concentration and depth for rain initiation, J. Geophys. Res.-Atmos., 117, D02207, https://doi.org/10.1029/2011JD016457, 2012.

Frisch, A. S., Fairall, C. W., and Snider, J. B.: Measurement of Stratus Cloud and Drizzle Parameters in ASTEX with a $\mathrm{K}_{\alpha}$-Band Doppler Radar and a Microwave Radiometer, J. Atmos. Sci., 52, 2788-2799, https://doi.org/10.1175/15200469(1995)052<2788:MOSCAD>2.0.CO;2, 1995a.

Frisch, A. S., Lenschow, D. H., Fairall, C. W., Schubert, W. H., and Gibson, J. S.: Doppler Radar Measurements of Turbulence in Marine Stratiform Cloud during ASTEX, J. Atmos. Sci., 52, 2800-2808, https://doi.org/10.1175/15200469(1995)052<2800:DRMOTI>2.0.CO;2, 1995b.

Gerber, H.: Microphysics of Marine Stratocumulus Clouds with Two Drizzle Modes, J. Atmos. Sci., 53, 1649-1662, https://doi.org/10.1175/1520 0469(1996)053<1649:MOMSCW>2.0.CO;2, 1996.

Giangrande, S. E., Babb, D. M., and Verlinde, J.: Processing Millimeter Wave Profiler Radar Spectra, J. Atmos. Ocean. Tech., 18, 1577-1583, https://doi.org/10.1175/15200426(2001)018<1577:PMWPRS>2.0.CO;2, 2001. 
Gonçalves, F. L. T., Martins, J. A., and Silva Dias, M. A.: Shape parameter analysis using cloud spectra and gamma functions in the numerical modeling RAMS during LBA Project at Amazonian region, Brazil, Atmos. Res., 89, 1-11, https://doi.org/10.1016/j.atmosres.2007.12.005, 2008.

Hartmann, D. L., Ockert-Bell, M. E., and Michelsen, M. L.: The Effect of Cloud Type on Earth's Energy Balance: Global Analysis, J. Climate, 5, 1281-1304, https://doi.org/10.1175/15200442(1992)005<1281:TEOCTO >2.0.CO;2, 1992.

Heus, T., van Heerwaarden, C. C., Jonker, H. J. J., Pier Siebesma, A., Axelsen, S., van den Dries, K., Geoffroy, O., Moene, A. F., Pino, D., de Roode, S. R., and Vilà-Guerau de Arellano, J.: Formulation of the Dutch Atmospheric Large-Eddy Simulation (DALES) and overview of its applications, Geosci. Model Dev., 3, 415-444, https://doi.org/10.5194/gmd-3-415-2010, 2010.

Hogan, R. J.: Fast approximate calculation of multiply scattered lidar returns, Appl. Opt., 45, 5984-5992, https://doi.org/10.1364/AO.45.005984, 2006.

Hogan, R. J.: Fast Lidar and Radar Multiple-Scattering Models. Part I: Small-Angle Scattering Using the Photon Variance-Covariance Method, J. Atmos. Sci., 65, 3621-3635, https://doi.org/10.1175/2008JAS2642.1, 2008.

Illingworth, A. J., Hogan, R. J., O'Connor, E. J., Bouniol, D., Brooks, M. E., Delanoë, J., Donovan, D. P., Eastment, J. D., Gaussiat, N., Goddard, J. W. F., Haeffelin, M., Baltink, H. K., Krasnov, O. A., Pelon, J., Piriou, J.-M., Protat, A., Russchenberg, H. W. J., Seifert, A., Tompkins, A. M., van Zadelhoff, G.-J., Vinit, F., Willén, U., Wilson, D. R., and Wrench, C. L.: Cloudnet: Continuous Evaluation of Cloud Profiles in Seven Operational Models Using Ground-Based Observations, B. Am. Meteorol. Soc., 88, 883-898, https://doi.org/10.1175/BAMS-88-6883, 2007.

Klett, J. D.: Stable analytical inversion solution for processing lidar returns, Appl. Opt., 20, 211-220, https://doi.org/10.1364/AO.20.000211, 1981.

Kollias, P., RéMillard, J., Luke, E., and Szyrmer, W.: Cloud radar Doppler spectra in drizzling stratiform clouds: 1. Forward modeling and remote sensing applications, J. Geophys. Res.-Atmos., 116, D13201, https://doi.org/10.1029/2010JD015237, 2011a.

Kollias, P., Szyrmer, W., RéMillard, J., and Luke, E.: Cloud radar Doppler spectra in drizzling stratiform clouds: 2. Observations and microphysical modeling of drizzle evolution, J. Geophys. Res.-Atmos., 116, D13203, https://doi.org/10.1029/2010JD015238, 2011b.

Kovalev, V. A.: Sensitivity of the lidar solution to errors of the aerosol backscatter-to-extinction ratio: influence of a monotonic change in the aerosol extinction coefficient, Appl. Opt., 34, 3457-3462, https://doi.org/10.1364/AO.34.003457, 1995.

Liebe, H. J., Hufford, G. A., and Manabe, T.: Millimeter-wave attenuation and delay rates due to fog/cloud conditions, IEEE T. Antenn. Propag., 37, 1617-1623, https://doi.org/10.1109/8.45106, 1989.

Liebe, H. J., Hufford, G. A., and Cotton, M. G. (Eds.): Propagation modeling of moist air and suspended water/ice particles at frequencies below $1000 \mathrm{GHz}$, AGARD Conference Proceeding, 1993.

Liu, Y., Geerts, B., Miller, M., Daum, P., and McGraw, R.: Threshold radar reflectivity for drizzling clouds, Geophys. Res. Lett., 35, L03807, https://doi.org/10.1029/2007GL031201, 2008.
Löhnert, U., Crewell, S., Simmer, C., and Macke, A.: Profiling Cloud Liquid Water by Combining Active and Passive Microwave Measurements with Cloud Model Statistics, J. Atmos. Ocean. Tech., 18, 1354-1366, https://doi.org/10.1175/15200426(2001)018<1354:PCLWBC>2.0.CO;2, 2001.

Lu, M.-L., Sorooshian, A., Jonsson, H. H., Feingold, G., Flagan, R. C., and Seinfeld, J. H.: Marine stratocumulus aerosol-cloud relationships in the MASE-II experiment: Precipitation susceptibility in eastern Pacific marine stratocumulus, J. Geophys. Res.Atmos., 114, D24203, https://doi.org/10.1029/2009JD012774, 2009.

Luke, E. P. and Kollias, P.: Separating Cloud and Drizzle Radar Moments during Precipitation Onset Using Doppler Spectra, J. Atmos. Ocean. Tech., 30, 1656-1671, https://doi.org/10.1175/JTECH-D-11-00195.1, 2013.

Mace, G. G. and Sassen, K.: A constrained algorithm for retrieval of stratocumulus cloud properties using solar radiation, microwave radiometer, and millimeter cloud radar data, J. Geophys. Res., 105, 29099-29108, https://doi.org/10.1029/2000JD900403, 2000.

Magaritz, L., Pinsky, M., Krasnov, O., and Khain, A.: Investigation of Droplet Size Distributions and Drizzle Formation Using A New Trajectory Ensemble Model. Part II: Lucky Parcels, J. Atmos. Sci., 66, 781-805, https://doi.org/10.1175/2008JAS2789.1, 2009.

Martucci, G. and O'Dowd, C. D.: Ground-based retrieval of continental and marine warm cloud microphysics, Atmos. Meas. Tech., 4, 2749-2765, https://doi.org/10.5194/amt-4-2749-2011, 2011.

McFarlane, S. A., Evans, K. F., and Ackerman, A. S.: A Bayesian algorithm for the retrieval of liquid water cloud properties from microwave radiometer and millimeter radar data, J. Geophys. Res.-Atmos., 107, AAC 12-1-AAC 12-21, https://doi.org/10.1029/2001JD001011, 2002.

Miles, N. L., Verlinde, J., and Clothiaux, E. E.: Cloud Droplet Size Distributions in Low-Level Stratiform Clouds, J. Atmos. Sci., 57, 295-311, https://doi.org/10.1175/15200469(2000)057<0295:CDSDIL>2.0.CO;2, 2000.

Myagkov, A., Seifert, P., Wandinger, U., Bühl, J., and Engelmann, R.: Relationship between temperature and apparent shape of pristine ice crystals derived from polarimetric cloud radar observations during the ACCEPT campaign, Atmos. Meas. Tech., 9, 3739-3754, https://doi.org/10.5194/amt-9-3739-2016, 2016.

O'Connor, E. J., Hogan, R. J., and Illingworth, A. J.: Retrieving Stratocumulus Drizzle Parameters Using Doppler Radar and Lidar, J. Appl. Meteorol., 44, 14-27, https://doi.org/10.1175/JAM2181.1, 2005.

Peter, R. and Kämpfer, N.: Radiometric determination of water vapor and liquid water and its validation with other techniques, J. Geophys. Res., 97, 18173-18183, https://doi.org/10.1029/92JD01717, 1992.

Pfitzenmaier, L., Dufournet, Y., Unal, C. M. H., and Russchenberg, H. W. J.: Retrieving Fall Streaks within Cloud Systems Using Doppler Radar, J. Atmos. Ocean. Tech., 34, 905-920, https://doi.org/10.1175/JTECH-D-16-0117.1, 2017.

Pinsky, M. B. and Khain, A. P.: Effects of in-cloud nucleation and turbulence on droplet spectrum formation in cumulus clouds, Q. J. Roy. Meteor. Soc., 128, 501-533, https://doi.org/10.1256/003590002321042072, 2002. 
Ramanathan, V., Cess, R. D., Harrison, E. F., Minnis, P., Barkstrom, B. R., Ahmad, E., and Hartmann, D.: CloudRadiative Forcing and Climate: Results from the Earth Radiation Budget Experiment, Science, 243, 57-63, https://doi.org/10.1126/science.243.4887.57, 1989.

Rosenfeld, D.: Suppression of Rain and Snow by Urban and Industrial Air Pollution, Science, 287, 1793-1796, https://doi.org/10.1126/science.287.5459.1793, 2000.

Rosenfeld, D. and Gutman, G.: Retrieving microphysical properties near the tops of potential rain clouds by multispectral analysis of AVHRR data, Atmos. Res., 34, 259-283, https://doi.org/10.1016/0169-8095(94)90096-5, 1994.

Rosenfeld, D., Wang, H., and Rasch, P. J.: The roles of cloud drop effective radius and LWP in determining rain properties in marine stratocumulus, Geophys. Res. Lett., 39, L13801, https://doi.org/10.1029/2012GL052028, 2012.

Rosenkranz, P. W.: Water vapor microwave continuum absorption: A comparison of measurements and models, Radio Sci., 33, 919 928, https://doi.org/10.1029/98RS01182, 1998.

Sauvageot, H. and Omar, J.: Radar Reflectivity of Cumulus Clouds, J. Atmos. Ocean. Tech., 4, 264-272, https://doi.org/10.1175/15200426(1987)004<0264:RROCC>2.0.CO;2, 1987.

Slingo, A.: Sensitivity of the Earth's radiation budget to changes in low clouds, Nature, 343, 49-51, https://doi.org/10.1038/343049a0, 1990.

Storn, R. and Price, K.: Differential Evolution - A Simple and Efficient Heuristic for Global Optimization over Continuous Spaces, J. Global Optim., 11, 341-359, https://doi.org/10.1023/A:1008202821328, 1997.

Suzuki, K., Nakajima, T. Y., and Stephens, G. L.: Particle Growth and Drop Collection Efficiency of Warm Clouds as Inferred from JointCloudSatand MODIS Observations, J. Atmos. Sci., 67, 3019-3032, https://doi.org/10.1175/2010JAS3463.1, 2010.
vanZanten, M. C., Stevens, B., Vali, G., and Lenschow, D. H.: Observations of Drizzle in Nocturnal Marine Stratocumulus, J. Atmos. Sci., 62, 88-106, https://doi.org/10.1175/JAS-3355.1, 2005.

Voors, R., Donovan, D., Acarreta, J., Eisinger, M., Franco, R., Lajas, D., Moyano, R., Pirondini, F., Ramos, J., and Wehr, T.: ECSIM: the simulator framework for EarthCARE, in: Sensors, Systems, and Next-Generation Satellites XI, 6744, SPIE Proceedings, 67441Y, https://doi.org/10.1117/12.737738, 2007.

Walko, R. L., Cotton, W. R., Meyers, M. P., and Harrington, J. Y.: New RAMS cloud microphysics parameterization part I: the single-moment scheme, Atmos. Res., 38, 29-62, https://doi.org/10.1016/0169-8095(94)00087-T, 1995.

Wang, J. and Geerts, B.: Identifying drizzle within marine stratus with W-band radar reflectivity, Atmos. Res., 69, 1-27, https://doi.org/10.1016/j.atmosres.2003.08.001, 2003.

Westbrook, C. D., Hogan, R. J., O'Connor, E. J., and Illingworth, A. J.: Estimating drizzle drop size and precipitation rate using two-colour lidar measurements, Atmos. Meas. Tech., 3, 671681, https://doi.org/10.5194/amt-3-671-2010, 2010.

Westwater, E. R.: The accuracy of water vapor and cloud liquid determination by dual-frequency groundbased microwave radiometry, Radio Sci., 13, 677-685, https://doi.org/10.1029/RS013i004p00677, 1978.

Wood, R.: Drizzle in Stratiform Boundary Layer Clouds. Part I: Vertical and Horizontal Structure, J. Atmos. Sci., 62, 3011-3033, https://doi.org/10.1175/JAS3529.1, 2005a.

Wood, R.: Drizzle in Stratiform Boundary Layer Clouds. Part II: Microphysical Aspects, J. Atmos. Sci., 62, 3034-3050, https://doi.org/10.1175/JAS3530.1, 2005b. 